\title{
Bolometric Voltage and Current (Bolovac) Standard for High and Microwave Frequencies
}

\author{
M. C. Selby* \\ Institute for Basic Standards, National Bureau of Standards, Boulder, Colo. 80302 \\ (October 31, 1967)
}

\begin{abstract}
A standard of voltages and currents for lumped-constant, coaxial, and other circuits operating in the TEM mode has been developed. Its approximate potential ranges are $1 \mathrm{MHz}$ to $20 \mathrm{GHz}$ in frequency, 0.05 to 10 volts, and 5 microamperes to 10 amperes. The principle may be used with bolometric, thermoelectric, photoelectric or other sensing elements consisting of thin film conducting or semiconducting disks located in the transverse plane of the TEM mode. Application and advantages are described and a comprehensive error analysis is presented. Figures illustrating construction details of one of the models of the Bolovac are included.
\end{abstract}

Key Words: Current measurement, measurement voltages, microwave current, microwave voltages, transmission-line voltages, voltage measurements.

\section{Introduction}

The first National Bureau of Standards (NBS) equipment to standardize sinusoidal voltages at frequencies up to $1 \mathrm{GHz}$ employed a two-thermistor-bead bolometer head of special design. It was developed at the NBS $[1]^{1}$ in the middle forties and was reproduced in other laboratories in the USA and abroad. A dynamometer type standard for $\mathrm{rf}$ current was also developed at the NBS; its principle and theory was analyzed [2] and an experimental model was built years ago. The construction of an improved instrument was recentlv completed [3]; a similar instrument, based on the same principles, was also built and put into operation in Japan.

The original NBS voltage standard has a frequency range of about 1 to $1000 \mathrm{MHz}$, a voltage range of about $20 \mathrm{mV}$ to $1.5 \mathrm{~V}$, and an approximate range of uncertainties of 0.3 to 2 percent. The rf current standard has a frequency range of about 1 to $1000 \mathrm{MHz}$, a current range of about 0.5 to $100 \mathrm{~A}$, and a range of uncertainties of 0.3 to 1 percent.

A new type bolometer head was developed at the NBS within the last several years for both voltage and current standardization having a considerably higher upper frequency and wider dynamic ranges at accuracies equal and potentially exceeding those above. This head will be described here and will be referred to as the "Bolometric Voltage and Current Standardization Head" or "Bolovac" for brevity. The basic principle and potential capabilities of the Bolovac

\footnotetext{
${ }^{*}$ Radio Standards Engineering Division, Boulder, Colo. 80302.

${ }^{1}$ Figures in brackets indicate the literature references at the end of this paper.
}

will be discussed here in the front sections. The following sections will indicate the critical elements of the device and the results obtained with the first models. The error analyses in this paper are generally limited to first order errors and uncertainties; higher order uncertainties are reserved for future analytical study and experimental verifications employing improved structural features of the Bolovac. Sources of overlooked uncertainties and possible means of their determination or elimination will be briefly discussed in the appropriate sections.

The Bolovac may be described as a source of known voltage and a sink of a known amount of current. It employs a disk-type bolometer split along one of its diameters and placed in a cylindrical coaxial line. It presently has a frequency range up to $10 \mathrm{GHz}$, a voltage range of about 0.1 to $5 \mathrm{~V}$ and a current range of about 5 to $200 \mathrm{~mA}$. Its potential frequency and dynamic ranges are much wider; e.g., it can be used to $20 \mathrm{GHz}$ and higher, to $10 \mathrm{~V}$ and to $10 \mathrm{~A}$ or higher depending partly on frequency. The principal advantages of the Bolovac are listed below and its limitations are discussed and analyzed later in the text.

The principal advantages of the Bolovac are as follows:

It serves as a standard of both voltage and current.

It has the widest frequency range of any standard of its kind known to date.

It employs a new type bolometer, namely, a split disk or disk sectors; other types, e.g., solid disks, barretters and semiconductors may also be employed for certain frequency and dynamic ranges. The disk may be made of metal, metal oxide, or carbon, depend- 
ing upon the frequency range and accuracy desired. It may be a thin film deposited on a substrate or a relatively thick conducting disk supported by a substrate. These disks have a wider range of resistance (fractions of an ohm to $1,000 \Omega$ or higher) than the present day high-frequency thermistors (having an approximate range of 5 to $100 \Omega$ ). The disks generally have positive temperature coefficients of resistivity as against generally negative ones for thermistors; this reduces the danger of overloading the bolometers in the absence of current-limiting impedances in the biasing and rf sources. Because the rf impedance of these disks is essentially equal to their d-c resistance over a wide frequency range, the Bolovac assembly may be used for some pulsed voltage and current measurements, e.g., as bolometric detectors.

It has potentially wider voltage and current ranges than the previous standards; its ability to measure currents down to $5 \mathrm{~mA}$ or lower with the accuracies indicated is of particular importance in view of present needs.

It provides a tool to measure microwave power transmitted down a uniform line with a potential accuracy comparable to the best present calorimetric techniques.

It eliminates the tedious and expensive thermistor matching problem required with the previous standard head. Thermistors must be matched with rf applied; the Bolovac disk sections are matched only at $\mathrm{d}$-c.

It is relatively simple in construction, much more rugged, and more reliable than thermistors. It may be used with a number of types of available bolometer bridges and d-c or low frequency measuring instrumentation depending upon the desired accuracies.

The principle and construction can be used directly in ammeters, e.g., for measuring base currents in vertical antennas, etc. The split disk type Bolovac retains the advantage of the two-thermistor mount in that it eliminates measurement errors associated with single thermistor mounts; the latter require stubs or other equivalent isolation components in the d-c or audio biasing circuitry.

It is relatively simple to operate and maintain (by comparison, for example, with a dynamometer type instrument requiring nonvibrating supports and fine suspensions, or with mounts employing fragile thermistor beads with their l-mil diam leads).

\section{Principle and Analysis}

Consider a conductive-film disk placed in a plane perpendicular to the axis of a uniform cylindrical coaxial lossless line energized by a source as illustrated in figure la. It will be shown below that, when the film thickness is smaller than one-fifteenth of the depth of the current penetration for the material, the equivalent circuit of the disk and the line for all practical purposes is that shown in figure $1 \mathrm{~b}$. In $1 \mathrm{~b} V_{m}$ is the rf voltage across the disk and is equal to the product of the d-c resistance of the disk and the rf current through it. The disk impedance may be made essentially equal to its d-c resistance for frequencies to $20 \mathrm{GHz}$ and higher. Here $V_{m}$ is detected and measured employing the well-known bolometric technique; other techniques, e.g., thermoelectric or photoelectric techniques may also be used for this purpose. If we employ the bolometric technique, we measure the rf power dissipated in the disk and we can compute the rf voltage across it at the frequencies in question and with accuracies approaching those of $d$-c measurements. For an assumed, unperturbed TEM field in the line at $R_{m}$, the measurement of $V_{m}$ is not affected by any other combinations of network elements shunting $R_{m}$; one of these elements may be the imput impedance of a voltmeter under calibration. The rf current through the disk, $I_{m}$, is measured by making $Z_{r}$ much higher than $R_{m}$, in which case the transmission line current, $I_{i}$, approaches the value of $I_{m}$ as $Z_{r}$ approaches infinity.

The disk may be considered a coaxial-line section of length $d$ and characteristic impedance

$$
Z_{0_{m}}=\eta_{m} \frac{1}{2 \pi} \ln \frac{r_{1}}{r_{2}}
$$

where $\eta_{m}$ is the intrinsic impedance of the metallic or semiconducting medium having a permittivity $\epsilon_{m}$, permeability $\boldsymbol{\mu}_{m}$, and conductivity $\sigma_{m} ; r_{1}$ and $r_{2}$ are the respective large and small radii of the line conductors.

At all frequencies under consideration here for media such as copper, silver, nickel, tantalum, carbon, etc. $[4,5]$,

$$
\eta_{m}=\left(\frac{j \omega \mu_{m}}{\sigma_{m}+j \omega \epsilon_{m}}\right)^{1 / 2} \cong(1+j)\left(\frac{\omega \mu_{m}}{2 \sigma_{m}}\right)^{1 / 2}
$$

and the propagation constant

$$
\gamma_{m}=\left[j \omega \mu_{m}\left(\sigma_{m}+j \omega \epsilon_{m}\right)\right]^{1 / 2} \cong(1+j)\left(\frac{\omega \mu_{m} \sigma_{m}}{2}\right)^{1 / 2}
$$
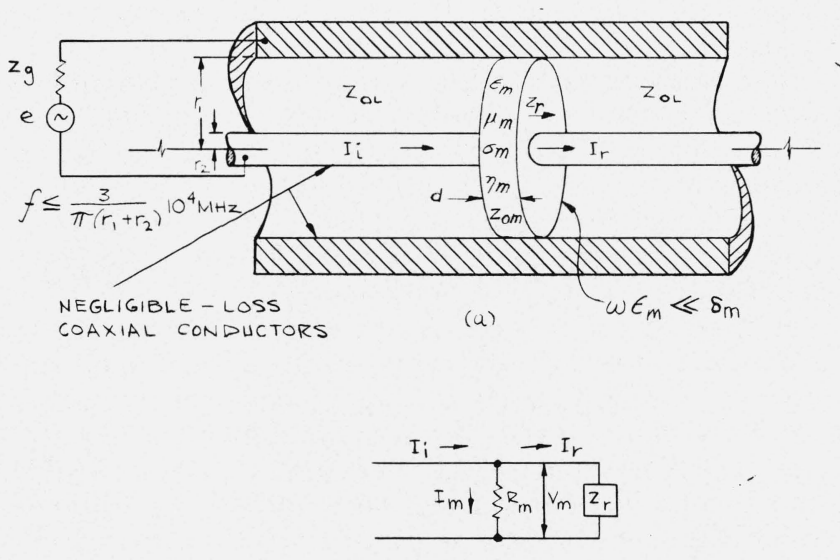

(b)

FiguRE 1. (a) Conductive-film disk placed in plane perpendicular to air-dielectric cylindrical coaxial line; $\mathrm{d} \ll \mathrm{r}_{2} ; \omega \epsilon \ll \sigma_{\mathrm{m}},($ b) equivalent circuit of la for skin depth $\delta_{\mathrm{m}} \geqslant 15 \mathrm{~d}$. 
because $\omega \epsilon_{m}$ (which has a value of 1.1 mhos per meter at $20 \mathrm{GHz}$ for copper for example) is negligible compared with $\sigma_{m}\left(5.8 \times 10^{7}\right.$ mhos per meter $)$.

Therefore

$$
Z_{o_{m}}=(1+j)\left(\frac{\omega \mu_{m}}{2 \sigma_{m}}\right)^{1 / 2} \frac{1}{2 \pi} \ln \frac{r_{1}}{r_{2}}
$$

The $\mathrm{d}$-c resistance of the disk is given by

$$
R_{m}=\frac{1}{2 \sigma_{m} \pi d} \ln \frac{r_{1}}{r_{2}}
$$

and the depth of current penetration into the solid metal of the film at a frequency $f$, is

$$
\delta_{m}=\left(\frac{1}{\sigma_{m} \pi f \mu_{m}}\right)^{1 / 2} .
$$

Substituting (5) and (6) into (4),

$$
Z_{0_{m}}=(1+j) R_{m} \frac{d}{\delta_{m}}
$$

and

$$
\gamma_{m}=(1+j) \frac{1}{\delta_{m}}
$$

From transmission line theory the output voltage, $V_{m}$, of the disk line section having a characteristic impedance of $Z_{0_{m}}$ is the product of the transfer impedance $Z_{t}$ and the input current $I_{i}$ :

$$
\begin{aligned}
Z_{t} & =\left(\frac{1}{Z_{r}} \cosh \gamma_{m} d+\frac{1}{Z_{o_{m}}} \sinh \gamma_{m} d\right)^{-1} \\
& =\left\{\frac{1}{Z_{r}} \cosh \left[(1+j) \frac{d}{\delta_{m}}\right]+\frac{1}{R_{m}} \frac{\sinh \left[(1+j) \frac{d}{\delta_{m}}\right]}{(1+j) \frac{d}{\delta_{m}}}\right\}^{-1} .
\end{aligned}
$$

Making use of available tables [6] one can show that for values of $\frac{d}{\delta_{m}} \leqslant 0.07$ we have in the worst case

$$
\begin{gathered}
(1+j) \frac{d}{\delta_{m}}=0.1 \underline{/ 45^{\circ}}, \\
\frac{\sinh \left(0.1 \underline{\left./ 45^{\circ}\right)}\right.}{0.1 \underline{/ 45^{\circ}}}=1.00000 \underline{/ 0.096^{\circ}} .
\end{gathered}
$$

$\operatorname{Cosh}\left(0.1 \underline{45^{\circ}}\right)=1.00001 \underline{10.287^{\circ}}$

Whence

$$
Z_{t}=\left[\frac{1}{Z_{r}}\left(1.00001 \underline{/ 0.3^{\circ}}\right)+\frac{1}{R_{m}}\left(1.00000 \underline{/ 0.1^{\circ}}\right)\right]^{-1} .
$$

Thus we see that for a ratio of $\frac{d}{\delta_{m}}=0.07$ one of the components of $Z_{t}$ will change in magnitude in terms of $\frac{1}{Z_{r}}$ by about 0.001 percent and will be shifted by $0.3^{\circ}$ in phase; the other component will remain essentially the same in magnitude and will be shifted in phase by $0.1^{\circ}$. These phase shifts are sufficiently small to be neglected; the magnitudes will be in error by less than 0.01 percent if we accept the value $R_{m}$ to be as shown in figure $1 b$, i.e.,

$$
Z_{t}=\left[\frac{1}{Z_{r}}+\frac{1}{R_{m}}\right]^{-1}=\frac{Z_{r} R_{m}}{Z_{r}+R_{m}}
$$

This indicates that the film need not be thinner than about one-fifteenth of the skin depth of the given metal at the maximum frequency of interest for a possible error of 0.01 percent from this cause. It will be shown below that in all practical cases this may be accomplished with a reasonable safety factor.

It also follows from (10) or from the equivalent circuit of figure $1 \mathrm{~b}$ that

$$
\text { for } Z_{r} \gg R_{m}, \quad I_{i} \cong \frac{V_{m}}{R_{m}} .
$$

A high $Z_{r}$ may be obtained by effectively terminating the line in a quarter wave section or in a parallel resonant LC circuit properly placed in the circuit.

One could arrive at eq (10) by simply showing that the disk impedance is resistive for $d \leqslant 0.07 \delta_{m}$; however, the more rigorous approach eliminates the question of the possible effect of the metal disk upon the TEM field distribution at the boundary planes of the air and metallic disk. It is not immediately apparent that the presence of the metallic disk will not perturb the radial electric field lines; one might be concerned with the capacity effect between the individual elements of the resistive disk and between each element of the disk and the transmission line conductors. One might also question the perturbation effect of the disk upon the concentric magnetic field lines because each element of the disk has its own external inductance.

The configuration of figure la can be treated as a composite of three cascaded transmission line sections of uniform diameters and infinite-conductivity conductors. This case has been analyzed in textbooks [7]. Results show that for coaxial-line inner and outer radii, $a$ and $b$, essentially no energy will be propagated along the line at modes other than the TEM at wavelength smaller than

$$
\lambda=\pi(b+a) .
$$

This approximate formula gives the critical frequency to within 8 percent. Furthermore, no higher modes can be produced by the presence of the film and substrate of the disk from the present TEM field; there are no longitudinal components of the electric and magnetic fields and the presence of the disk (the 
$Z_{0_{m}}$ section) does not introduce capacitive or inductive components into the system (except of those of its intrinsic characteristic impedance). This may not be the case when there are step discontinuities at the interfaces because of line diameter changes; the effect in this latter case will be discussed further on.

One may also heuristically show that the presence of the disk will not distort the field. This effect is negligible because of the circular and axial symmetry of the structure. For each electric and magnetic field line contributed by a given element of the disk, there is an equal and opposite field line contributed by another element located diametrically opposite the first, and the two cancel each other [8]. The effect of the substrate (insulator) upon which the metal film is deposited will also be discussed later on.

\section{Description of the Bolovac}

The heart of the Bolovac is a conductive bolometric film deposited on one face of an insulating disk (substrate). The film is split along one of the disk diameters, thus forming two bolometer elements of approximately semicircular or disk sector shapes. This disk is mounted in a specially constructed assembly permitting the following electrical connections: (1) the two bolometers are connected in series for the biasing energy and in parallel for the rf energy; (2) the plane where the standardizing rf voltage appears is located essentially at the input plane of the rf voltage indicating device being calibrated; (3) the plane through which the rf standardizing current is flowing is located essentially at the output plane of the current indicating device being calibrated. The distances from the rf-voltage or rf-current reference planes to the reference planes of the devices being calibrated is of the order of 1 to 2 mils. This distance is the thickness of the dielectric required to form a capacitance to isolate the biasing from the rf powers entering the Bolovac. Details of the disk and Bolovac assembly are shown in the figures below. The term "disk" below applies to the finished unit.

In principle the disk need not be split and the Bolovac may indeed be constructed with nonsplit disks. The relative advantages of splitting the disk are as follows. When bolometry is used, one generally needs to supply biasing power to the bolometer. Splitting the disk (dual element) eliminates the need of rf chokes or coaxial stubs required for a d-c return path. The d-c resistance of the chokes interferes with the precision and convenience of bridge operations. Stubs have to be tuned and require capacitors at the "shorted" end. Biasing currents should generally not be allowed in the rf current meters under calibration. Splitting the disk makes this possible. In case of the Bolovac, splitting the disk improves the bridge sensitivity generally by a factor of four. When the thermoelectric or photoelectric principle is used, generally no biasing power is needed, and solid disks may be preferable; however, the applications of these principles may introduce other difficulties partic- ularly in the choice of materials and fabrication of the disks.

Figure 2a shows the basic circuit of the Bolovac applied for $\mathrm{rf}$ voltage standardization, one of the possible biasing arrangements (in this case a d-c source) and a symmetrical measuring bridge. The analysis and computation are identical to those presented for the voltage standardization bridge employing thermistors [1].

The voltage, $V_{\text {rf }}$, applied to the unknown being calibrated is given for the symmetrical bridge by

$$
V_{\mathrm{rf}}=\frac{R_{T}}{2\left(R_{T}+R_{b}\right)}\left[\left(V_{R 2}-V_{R 1}\right)\left(2 V_{0}-V_{R 2}-V_{R 1}\right)\right]^{1 / 2} .
$$

Here $V_{R 1}$ and $V_{R 2}$ are the respective voltage drops across the adjustable resistance in series with the d-c source before and after rf power is applied to the Bolovac with the bridge balanced in both cases. $R_{T}$ and $R_{b}$ are the respective Bolovac and bridge arm resistances as shown in figure $2 \mathrm{a}$.

Figure $2 b$ shows a schematic of the circuit arrangement with the Bolovac used for current standardization. The basic difference between figures $2 a$ and $2 b$ is that in 2 a the standard $V_{\text {rf }}$ is obtained at the output terminals of the Bolovac, whereas in $2 \mathrm{~b}$ the standard current, $I_{\mathrm{rf}}$, is obtained at the input terminals to the Bolovac. The schematic shows a thermoelement being calibrated in terms of the rf current entering the Bolovac. A constant-current d-c biasing source is shown in this case [9] and $I_{\mathrm{rf}}$ is given for a symmetrical bridge by

$$
I_{r f}=\left[2 I_{s} I_{\mathrm{dco}}-I_{s}^{2}\right]^{1 / 2}
$$

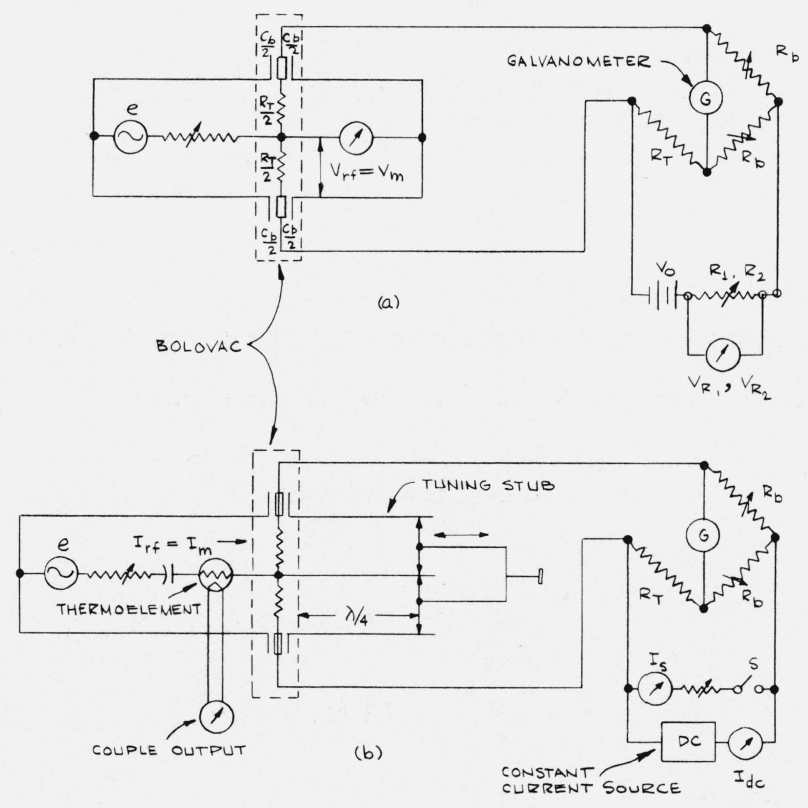

FIGURE 2. Schematic diagram showing the applications of the Bolovac for (a) voltage standardization, and (b) current standardization. 
Here $I_{d c o}$ is the $d$-c biasing current in the bridge at initial balance (without rf) for $I_{s}=$ zero, and $I_{s}$ is the amount by which the $I_{\text {dco }}$ was reduced for final balance (with rf) with switch $\mathrm{S}$ closed.

In both voltage and current cases it is assumed that the two sections of the Bolovac disk have been adjusted for equal resistance. Correction factors may be used if these resistances are not exactly alike; it will be shown later that this requirement is not critical. It is, of course, apparent that either of the two shown and other biasing arrangements may be used for both rf voltage and current standardization.

Figure 3 shows the top view and cross section of a Bolovac disk. The substrate of the disk may be made of any suitable insulating material such as quartz, Pyrex, or high thermal-conductivity ceramic (to allow higher current carrying capacity for the conducting film). Electrodes may be fired or evaporated on the cylindrical surfaces and, if necessary, at their rims to make sure of good contact with the conductors of the coaxial line system. A thin conducting film is then deposited on one of the flat surfaces. The radial gaps between the two halves of the circular film may be produced by a mask during evaporation or by removing parts of the film after evaporation. The gaps extend to the center electrode ring interconnecting these halves of the film in series for the biasing current and in parallel for the rf current as was pointed out above. As stated before, the film may

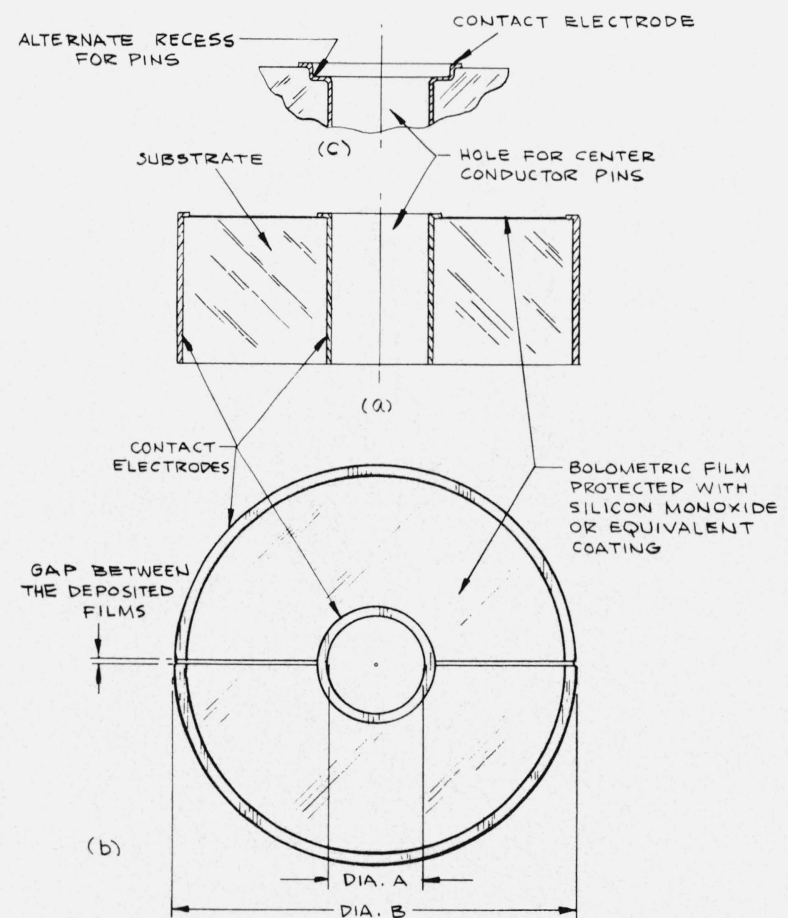

NOTE: FOR BEST RESLLTS DIAMETERS A \& B SHOLLD BE THE SAME AS THE CORRESPONDING DIAMETERS OF THE COAXIAL LINE SYSTEM WITH THE RESISTIVE FILM COVERING UNIFORMLY THE ENTIRE

Figure 3. Bolovac bolometric disk (a) cross-section, (b) top view, (c) alternate recess for pin to avoid a discontinuity. be made of noble metals, chromium, nickel, tantalum, metal oxides, or carbon, etc. The width of the annulus of the film should, as closely as possible, be equal to the spacing between the coaxial conductors to reduce step discontinuities to a minimum. One must be cautious with some materials for the films to make certain the capacities between the particles (sometimes referred to as the "Boella" effect) will not introduce a reactive component even with sufficiently thin films. It may, however, be possible to determine by measurement the departure of the value of $\mathrm{rf}$ resistance of the bolometer elements from their d-c values and introduce an appropriate correction in the computed values of the voltages or currents. The art of film and electrode deposition and of disk substrate fabrication has recently attained a degree of perfection sufficient for the objectives in question here. A brief appraisal of the limitations and likely errors from disk fabrication imperfections is given in the appendix. The first disks for the Bolovac were made at the NBS. The substrates were cut in the instrument shops and the electrodes and films were deposited employing sputtering or evaporation and firing. The most stable NBS disks to date had platinum films with power resistivity constants of the order of two ohms per watt. Parameters of the disk and their correlation to the performance results will be discussed in the section on error analysis.

The Bolovac consists of a core and an auxiliary rf bypassing capacitor when needed. The core has two disk-supporting semicircular or rectangular metal plates sandwiched between two other "ground" metal plates. Figures 4, 5, and 6 show the details of the core and capacitor ring assembly and are selfexplanatory. The bolometer disk is clamped, soldered, or cemented between two half disks forming an extension of the biasing electrodes; the clamping may be accomplished by means of plastic or insulated steel bolts. The insulation between the outside "ground" plates and the inside composite plate is the dielectric of the rf bypassing capacitors $C_{b}$ shown in figure 2; the outer plates are thus the common ground of the system for the rf power. Referring , to figure $4 \mathrm{a}$ the $\mathrm{rf}$ power is fed into the bottom plate and the standardizing voltage appears in the plane A of the bolometric film. The input plane to the voltmeter under calibration is in plane $\mathrm{A}^{\prime}$ a specified distance of 1 to 2 mils away from A. It is, therefore, imperative to design the disk pin and plate receptacle dimensions to maintain this spacing between the two planes, particularly for frequencies of $1 \mathrm{GHz}$ and higher. With the quality of rf connectors continuously improving, and especially with the new precision sexless connectors currently being standardized, this requirement should not present any serious problems.

Again, referring to figure $4 \mathrm{a}$, for current standardization, the top plate of the core may be replaced by another plate incorporating the enclosure for the current-measuring device to be calibrated. Or an adapter may be built to enable the connection of such a housing without removing the entire top plate; an example 


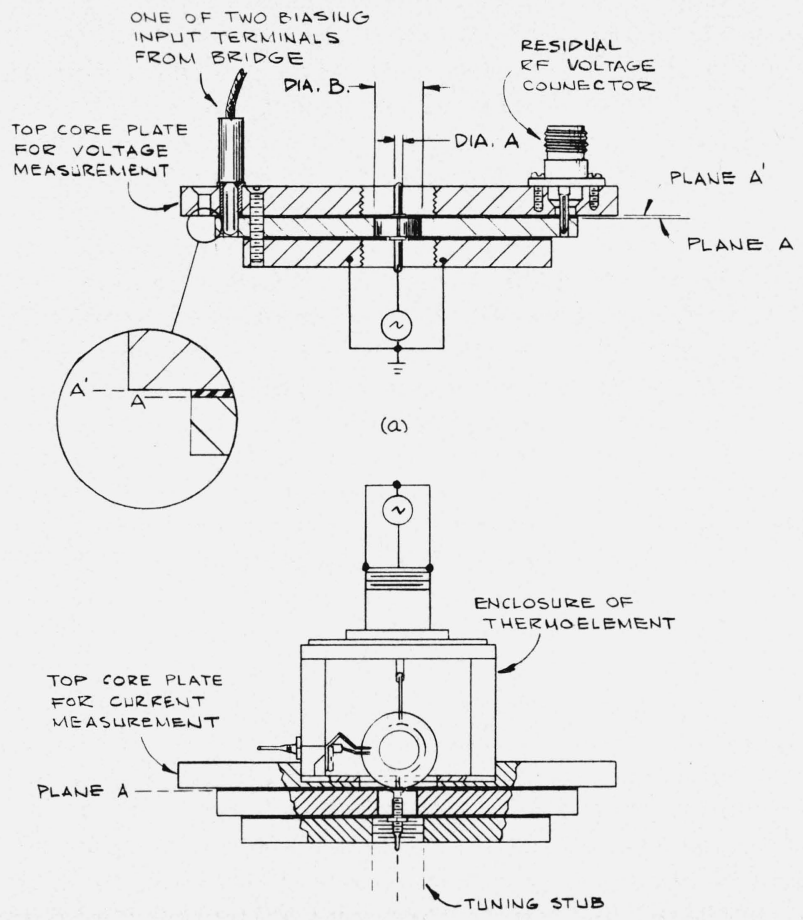

(b)

Figure 4. Structural components of the Bolovac; (a) core cross section for voltage measurement, $(b)$ core cross section for current measurement.

of such an adapter is shown in figure $4 \mathrm{~b}$. The top plate with one kind of an enclosure for a certain type of thermoelement is shown in this figure. The rf current enters the core via the input into the housing and the standardizing current crosses reference plane $A$ in which the film is located. The output reference plane of the current-sensor in its housing is thus essentially in plane A irrespective of how far the thermoelement is actually located from A. One must, however, reproduce this reference plane $A$ when the enclosure of the "current meter" is removed from the Bolovac and put into use. The effect of the impedance in plane A as seen by the "current meter" and of the mechanical discontinuity upon the TEM mode are discussed under error analysis. For current standardization, the "output" port of the core is used for attaching an adjustable shorting line, a low-capacity shielding cap, or a tuned LC tank to terminate the system in a high impedance $Z_{r}$ as discussed in the previous section. In some cases one may use a tube, equivalent to an outer conductor of the line, of sufficient length to prevent radiation from this end of the core. The latter may prove more economical than a shorting stub provided the value of the terminating impedance is high enough for the desired accuracy.

Figure 5 shows exploded views of the core assembly for voltage and current standardization.

The capacity $C_{b}$ of the core itself (see fig. 2) is of the order of $2000 \mathrm{pF}$ between each outer ground plate and the composite inner plate. In order to keep the effective impedance of $C_{b}$ at a value sufficiently low

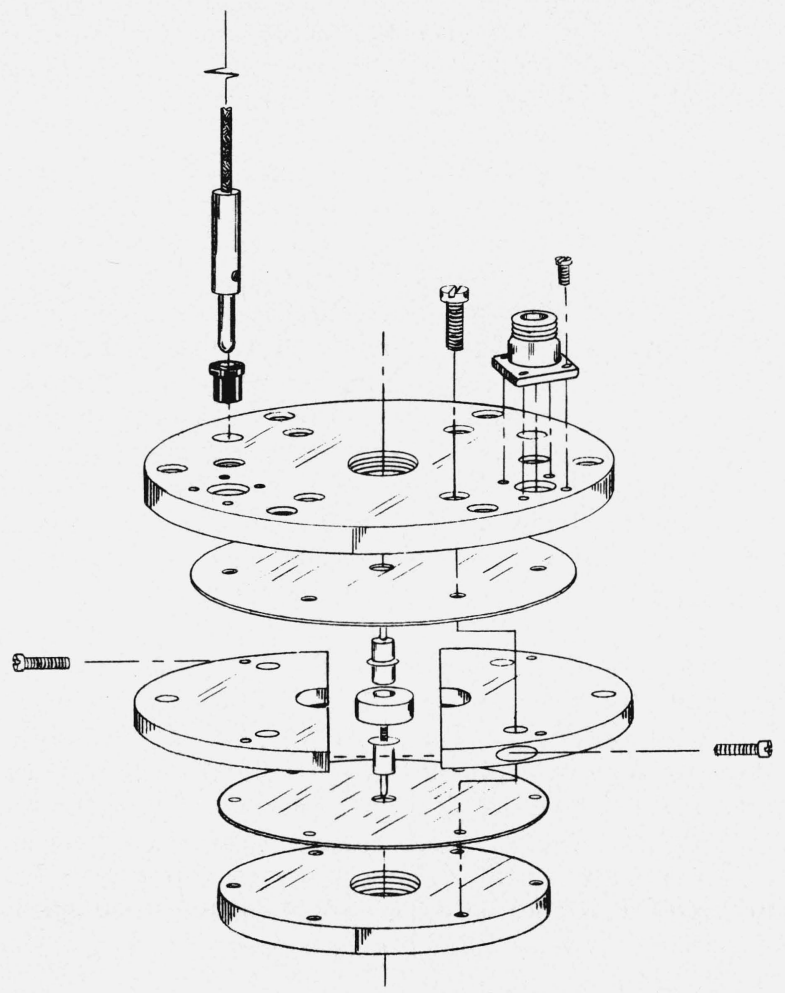

FIGURE 5a. Exploded view of core for voltage standardization.

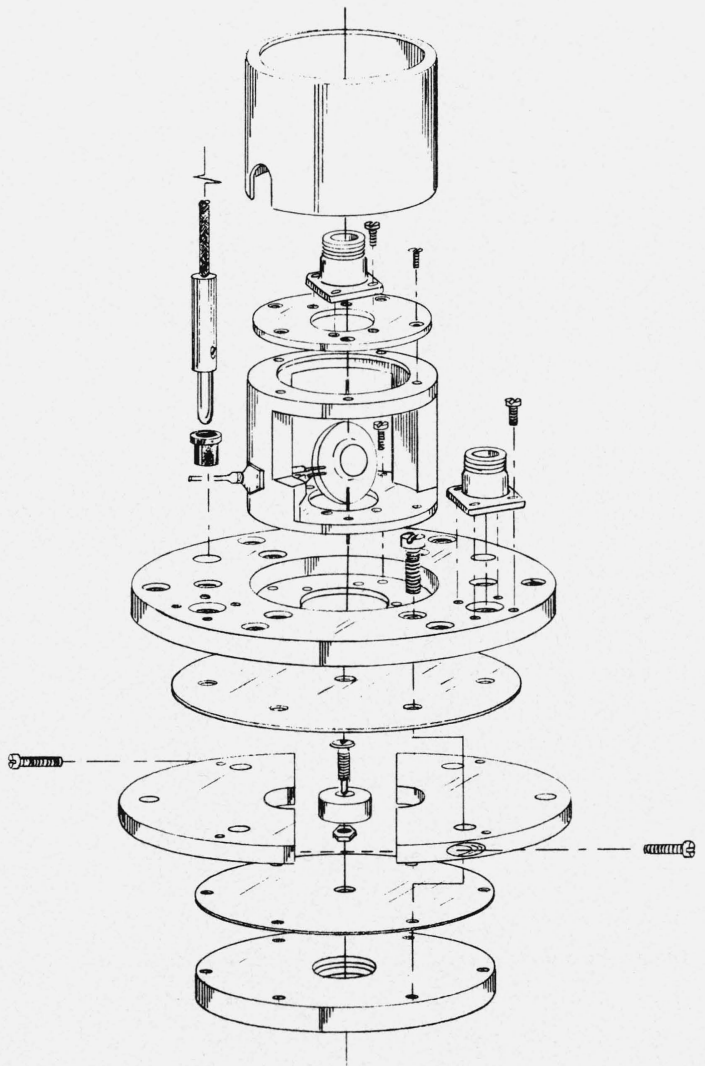

FiguRE 5b. Exploded view of core for current standardization. 
so that the rf voltage drop across it does not introduce serious errors, particularly at low frequencies (discussed under error analysis), the additional ringshaped capacitor mentioned above was constructed; this ring is generally not needed for current measurements. It consists of additional ground and center plates with a large number of fixed capacitors imbedded into the center plates. Thus, the value of $C_{b}$ is increased to about $60,000 \mathrm{pF}$. The construction details are shown in figure 6 . One may use the core with or without the ring depending on the frequency. Other structures are, of course, possible in order to increase the value of $C_{b}$ of the core and ring, and one may increase it further by adding fixed capacitors, for example, at the outside rim of the ring. The application of anodized aluminum plates or other similar techniques may be considered to obtain the largest value of $C_{b}$ per unit volume and weight of the structure.

Type N rf connectors on the two sides of the core (fig. 4a) may be used for measuring the biasing potentials as well as for probing the rf voltage mentioned above across $C_{b}$ when wanted.

Figure 6 shows an exploded assembly of $C_{b}$ and figure 7 shows a perspective view of the assembled core with the voltage plate and the capacitor ring. Figure 8 shows the voltage and current center conductor pins used with the disk. Figures 9a, b, etc., show photographs of the components and assemblies de-

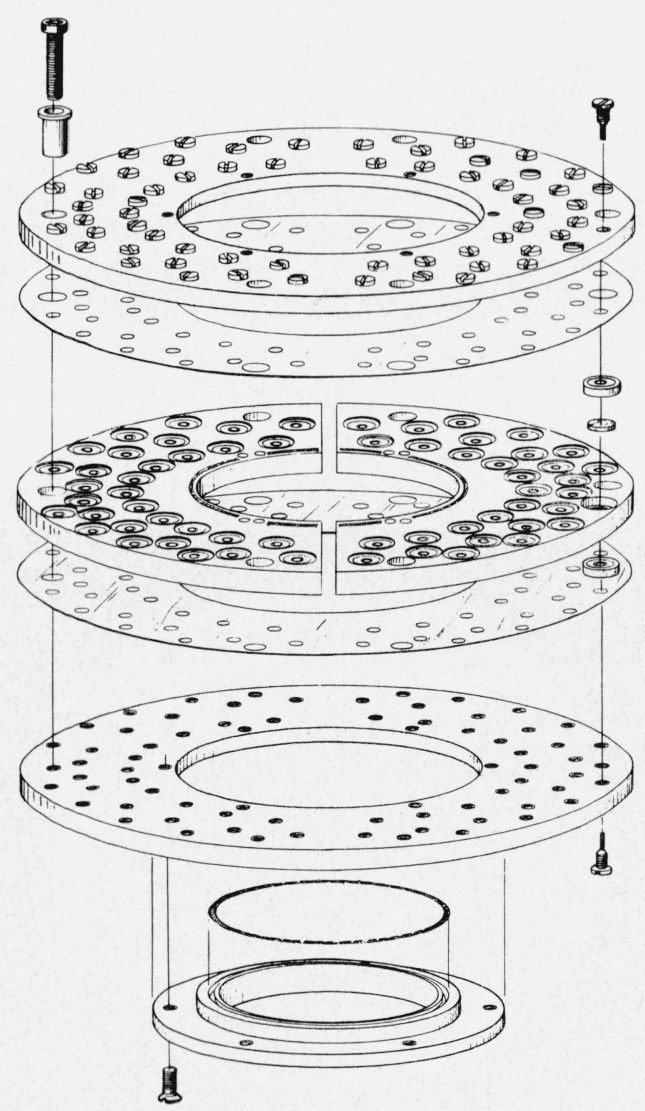

FIGURE 6. Exploded view of ring-shaped auxiliary capacitor.

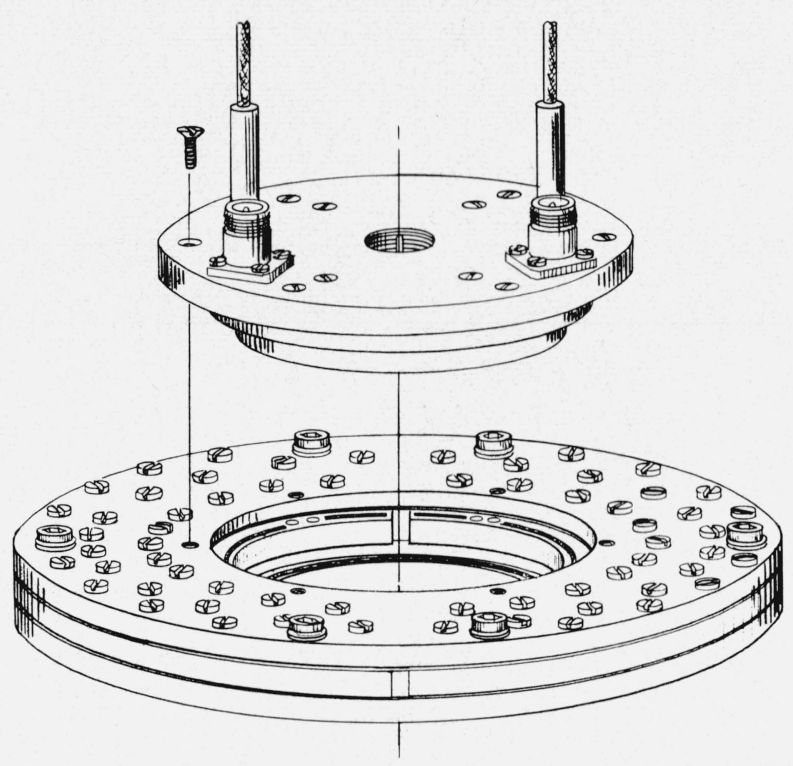

FIGURE 7. Core (with voltage plate) and auxiliary capacitor of Bolovac.

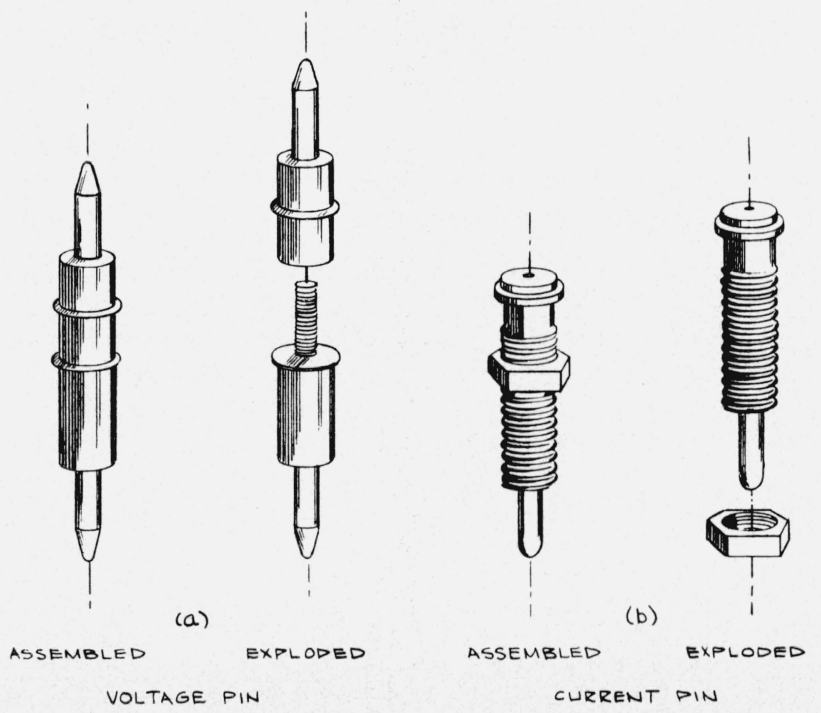

Figure 8. Center conductor pins of Bolovac disk; (a) for voltage measurement, (b) for current measurement.

scribed above. The auxiliary $C_{b}$ assembly may, of course, have shapes other than the ring type used here.

\section{Results and Error Analysis}

\subsection{Results}

Most of the measurements were made at voltage and current levels at which intercomparisons with power standards in a $50-\Omega$ system could be made directly (without auxiliary attenuation or amplification). Calorimetric power measurements served as 


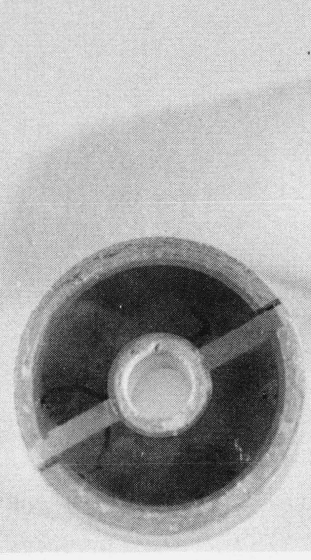

FIGURE 9a. Bolovac split-film resistors and substrates (disks).

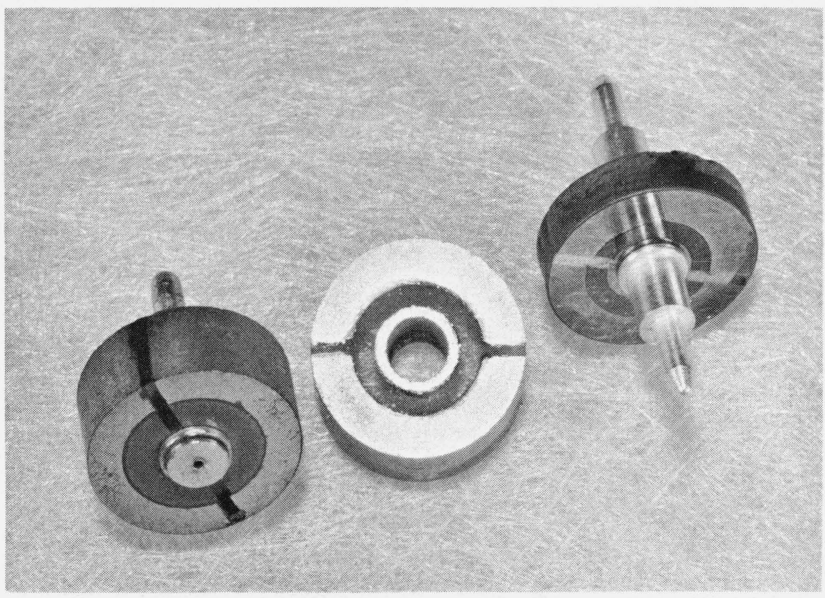

Figure 9b. Disks without and with voltage and current coaxconductor pins.

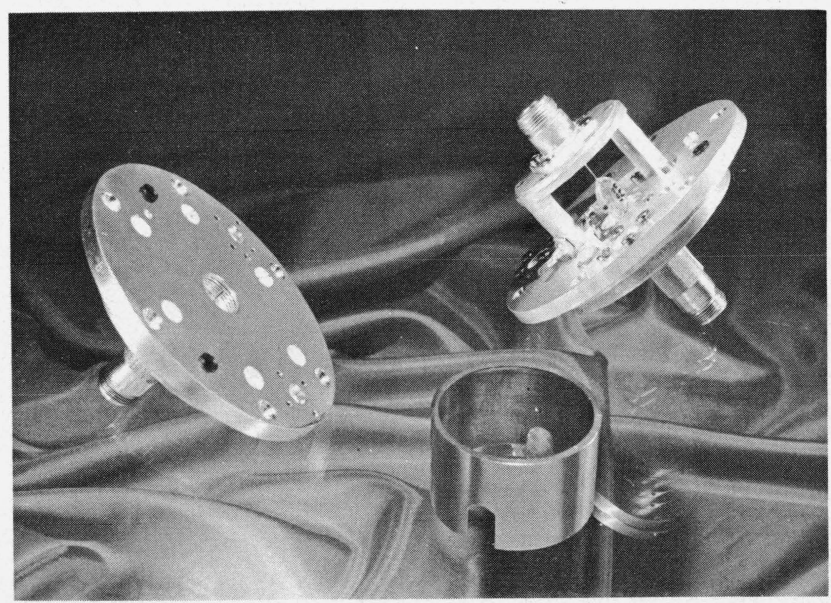

Figure 9c. Voltage and current cores assembled.

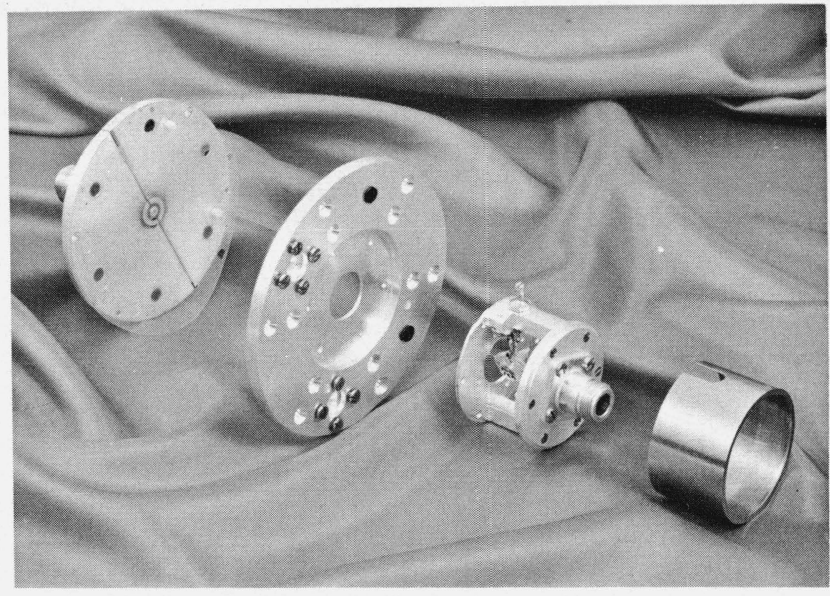

Figure 9d. Current core exploded.

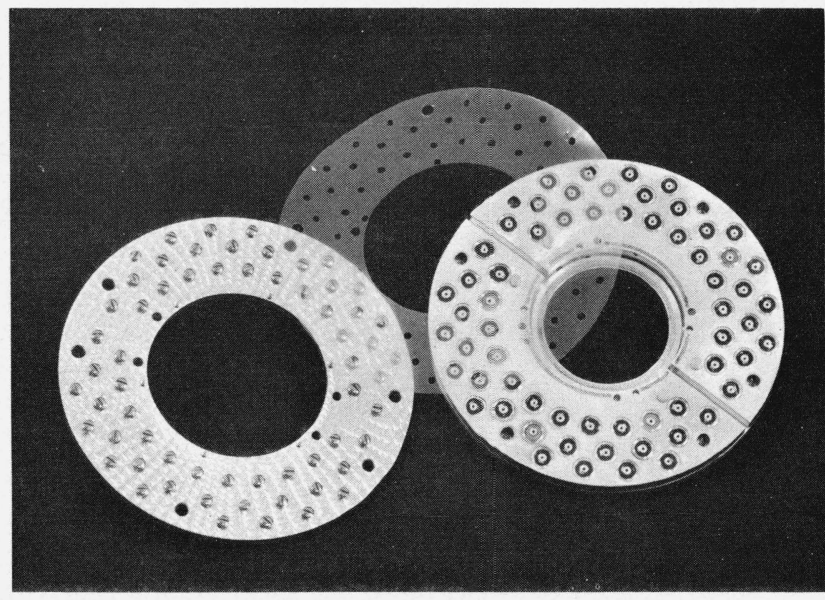

FIGURE 9e. Auxiliary capacitor with top plate and insulator removed.

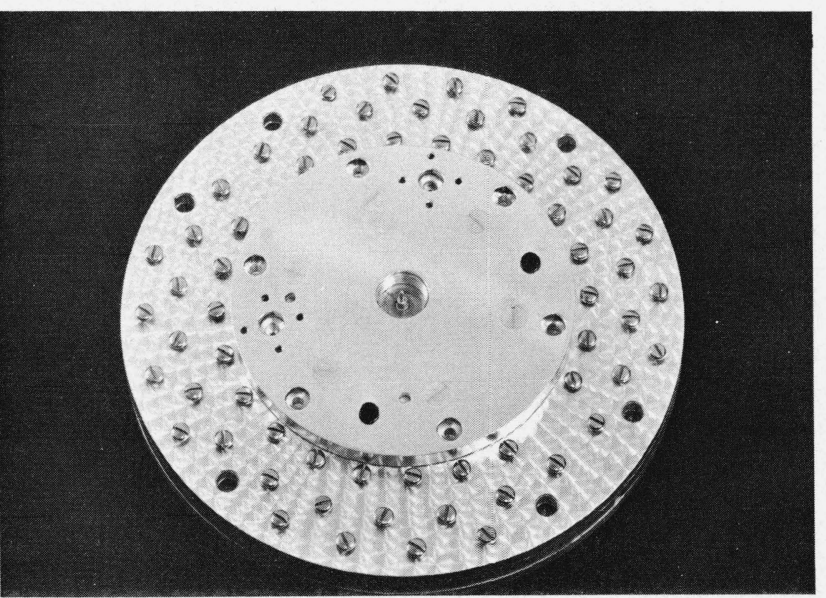

Figure 9f. Voltage core and auxiliary capacitor. 


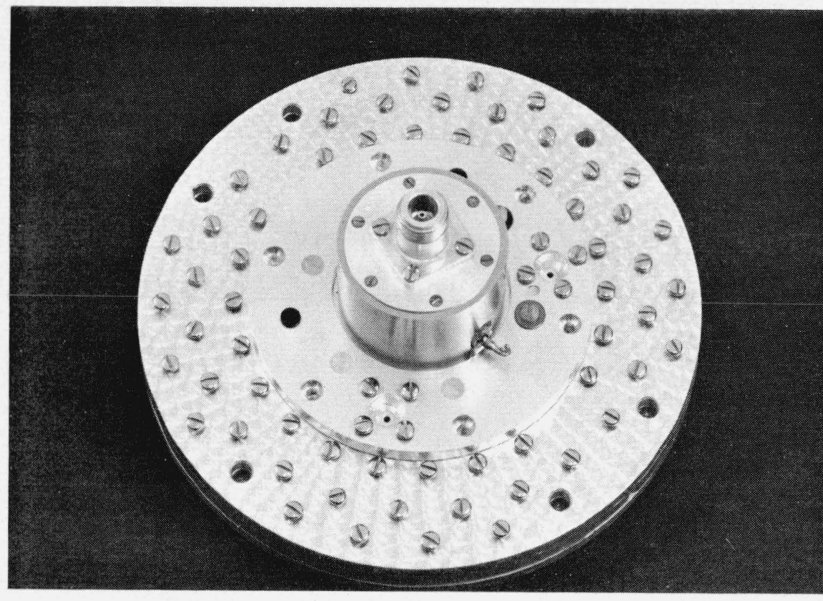

Figure 9g. Current core and auxiliary capacitor.

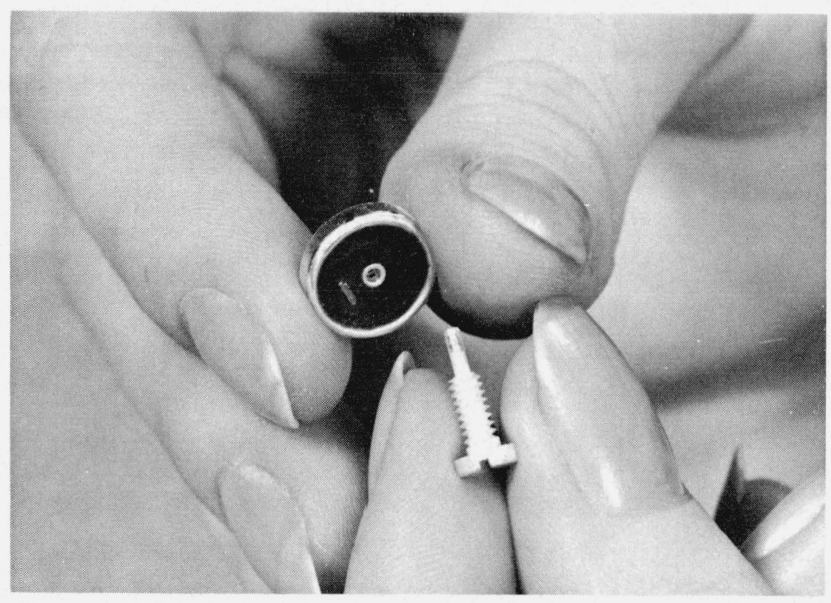

FIGURE 9h. Capacitor disk and grounding-electrode bolt (beryllium copper split contact pin).

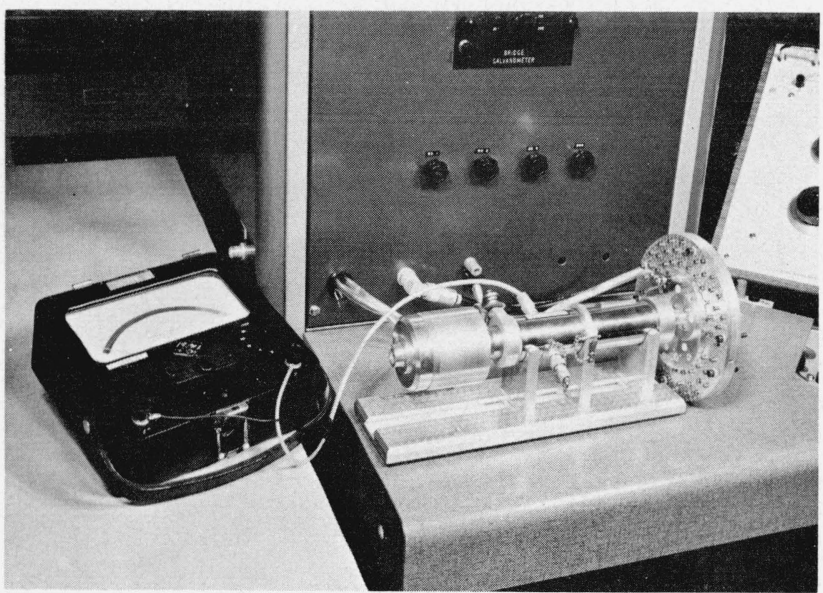

Figure 9i. Bolovac set up to calibrate ATVM.

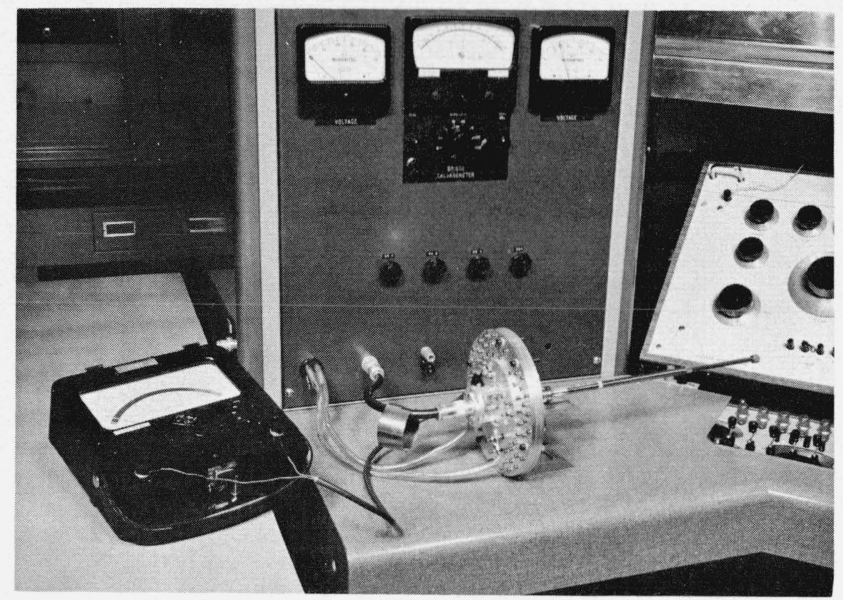

FigURE 9j. Bolovac set up to calibrate thermoelement.

independent direct or indirect comparisons for both voltage and current. Intercomparisons with thermistor mounts at frequencies to $1 \mathrm{GHz}$ and between a number of Bolovac cores and disks above $1 \mathrm{GHz}$ served as quasi-independent techniques; the latter furnished confidence in the quality of the disks and the assemblies of the system as a whole. In addition, intercomparisons were made employing two independent bridge consoles, one a constant dc-voltage biasing type, the other with constant dc-current biasing. The agreement at voltage levels of about 0.2 to $1.5 \mathrm{~V}$ was 1 percent or better to $2 \mathrm{GHz}, 2$ percent at $4 \mathrm{GHz}$ and 4 percent at $8 \mathrm{GHz}$. The agreement in measuring currents of 5 to $75 \mathrm{~mA}$ (in 5 to $200 \mathrm{~mA}$ thermoelements) was 1 percent or better to $1 \mathrm{GHz}$. Reproducibility of current measurements was within 1 percent at 2, 3, and $4 \mathrm{GHz}$; comparison with power was not made at these frequencies because of resonances exhibited by the thermoelements, apparently as a result of residual inductances and shunt capacities of the heaters.

Figure 10 shows calibration curves of these thermoelements obtained, to our knowledge, for the first time over the frequency range and to the accuracies given above.

A conservative estimate of the uncertainties of the measured voltages and currents in terms of assumed true values is equal to the above figures on the agreements. It is felt that a several-fold improvement over the above results may be achieved because our efforts to date to perfect the Bolovac and the measuring system were at best only moderate.

To prevent misinterpretation of the following "error analysis" it is advisable to define terms as used here. An "error in mathematics" is defined as "the difference between the true value and the calculated or observed value" [10]. A "result" may be corrected for a given "error." Because the "true" value is never known, the absolute magnitude of the "error" can only be approximately determined. A result is known to an estimated plus or minus "uncertainty"; the result cannot be corrected for an "uncertainty." The "un- 


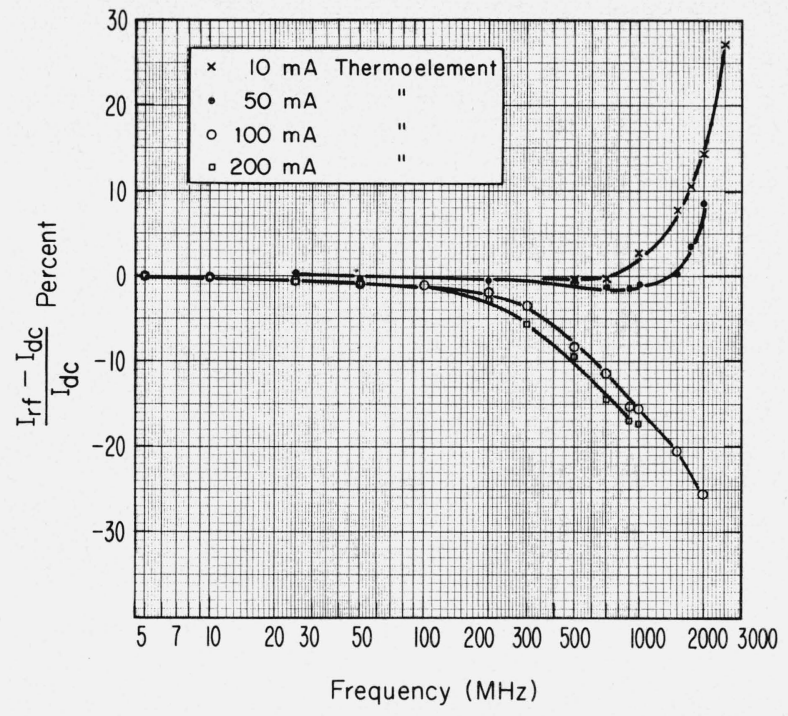

FIGURE 10. Calibration of the UHF thermoelements with Bolovac.

certainty" may consist of a number of uncertainties of parameter values entering an equation and includes the uncertainty in the "error." "Uncertainty" is often referred to as "limits of error."

The results of the technique described here are subject to the following sources of uncertainties:

Approximations in the analytical steps leading to eqs (10), (11), (12), and (13).

Uncertainty of values of bridge components, biasing source instability, limits of bridge sensitivity, balancing galvanometer noise, and rf source instability.

Quality of bolometric disk and its substrate.

Standing waves and discontinuities in the transmission line.

Input impedance of the resonant circuit or of quarter wave coaxial line section shunting $R_{m}$ for current measurements.

Residual impedances in the ground system.

An assessment of the uncertainties contributed by these sources follows.

\subsection{Effect of Approximations Leading to eqs (10) to (13)}

It was shown earlier that the magnitude of the voltage or current may be in error by less than 0.01 percent if the disk-film thickness, $d$, is 7 percent of the skin depth, $\delta_{m}$. In practice the ratio $\frac{d}{\delta_{m}}$ is always smaller than 0.07 .

The resistance, and the depth of current penetration into the annular resistor is given by $[4,5,11]$.

$$
\begin{gathered}
R_{m}=\frac{1.724 \times 10^{-8} \rho_{m} / \rho_{c}}{2 \pi d} \ln \frac{r_{1}}{r_{2}} \text { ohms } \\
\delta_{m}=\frac{0.0661}{f^{1 / 2}}\left(\frac{1}{\mu_{m r}}\right)^{1 / 2}\left(\frac{\rho_{m}}{\rho_{c}}\right)^{1 / 2} \text { meters }
\end{gathered}
$$

where $d$ is in meters, $f$ is in $\mathrm{Hz}, \mu_{m r}$ is relative permeability of the conductor $\left(\mu_{m r}=1\right.$ for copper and other nonmagnetic materials); $\rho_{m} / \rho_{c}=$ relative resistivity (in terms of copper at $20^{\circ} \mathrm{C}, \rho_{c}=1.724 \times 10^{-8}$ $\Omega \mathrm{m})$.

$$
\begin{aligned}
& \text { For } \frac{r_{1}}{r_{2}}=2.3\left(Z_{0}=50 \mathrm{ohms}\right), \\
& \qquad \frac{d}{\delta_{m}}=3.46 \times 10^{-8} \frac{1}{R_{m}}\left(\frac{\rho_{m}}{\rho_{c}} \mu_{m r} f\right)^{1 / 2} .
\end{aligned}
$$

Assuming an extreme pessimistic case of $R_{m}=0.1 \Omega$ at $20 \mathrm{GHz}$ for copper,

$$
d \cong 0.05 \delta_{m} .
$$

It follows from eq (16) that higher values of $R_{m}$ may be realized by using higher resistivity materials without changing the ratio of $\frac{d}{\delta_{m}}$. When materials of sufficient resistivity are not available, one may reduce the film thickness, $d$, in order to increase $R_{m}$. However, $d$ cannot be reduced beyond the point at which the film stops behaving as a solid conductor. When this limit is reached, one may have to compromise for higher ratios of $\frac{d}{\delta_{m}}$, and consequent narrower frequency range or possibly larger penetration error.

\subsection{Uncertainty Introduced by Bridge Assembly Components}

Uncertainties were introduced into the results by the random drift of the d-c power supply, the error in the values of the $d$-c resistors, the random drift and temperature coefficient of the d-c resistors during the time it took to make a measurement, the uncertainty in measuring d-c voltages before and after $\mathrm{rf}$ was applied to the bridge, and the d-c imbalance of the two halves of the disk resistors [12]. An estimate of the limiting uncertainty from these sources is \pm 0.06 percent. Another source of uncertainty was the limited stability of the rf sources during a measurement, which is estimated as \pm 0.01 percent. The outside limits of the uncertainties introduced by these causes are thus \pm 0.07 percent.

\subsection{Quality of Bolometric Disk and of Its Substrate}

Two parameters of the film and substrate assembly will affect the uncertainty of the results. The first is the film sensitivity designated by $\gamma_{m}$ (ohms/watt), the incremental resistance as a function of power dissipated in the disk. The second is the film stability which will affect the signal to noise ratio of the bridge galvanometer, particularly at low levels of $V_{m}$. As already stated, film and disk fabrication details are outside the scope of this paper. Such factors as the quality of the substrate surfaces, aging and protection of the films, migration of contact electrode metals into the film, uniformity of the film thickness, etc., 
have been apparently well under control and should contribute negligible uncertainty in the results. Some information on the state of the art of film deposition and of the uncertainties imposed by its limitations are discussed in the appendix. Thermal noise of the disks (of the order of $200 \mu \mathrm{V}$ for a bandwidth of $10 \mathrm{GHz}$ for a $200 \Omega$ disk at room temperature) may also be neglected at the voltage and current levels here under consideration, particularly for "single" frequency or narrow band applications.

However, the uncertainty is affected considerably by $\gamma_{m}$ because the bridge sensitivity (expressed as the galvanometer deflection for a given voltage or current increment in the disk) is directly proportional to $\gamma_{m}$ [12]. The results shown above were obtained with platinum film disks; their $\gamma_{m}$ values in the assembly were about 1 to $3 \Omega / \mathbb{W}$. Some chromium, nickel, and gold disks were also fabricated at the NBS without great improvement of $\gamma_{m}$; in one case of a $50 \Omega(200$ $\Omega$ d-c) nickel unit, $\gamma_{m}$ was $9 \Omega / \mathbb{W}$. Measurements of carbon films indicated a $\gamma_{m}$ of 2 to 7 . There is evidence that values of $10 \Omega / W$ and higher are obtainable in laboratories outside the NBS. Our platinum units were considerably more stable and had a much longer life than the chromium or nickel units. It seems rather difficult to predict the value of $\gamma_{m}$ because the temperature coefficient of the conductivity of films is a pronounced function of their thickness. The temperature coefficient is negative for values below about $100 \AA$, then crosses the zero line and becomes positive as the thickness increases [13]. For two disks having the same temperature coefficients of resistivity it seems more promising to use higher resistivity metals or alloys because a thicker film will be realized for a given disk resistance. This may insure greater stability.

There will be an error in the measured voltage or current if the resistances or the values of $\gamma_{m}$ of the two halves of the film-disks are not equal. This error has been discussed in the past $[1,12,14,15]$ and is approximately proportional to the product of (1) the fractional difference of the initial resistances, and (2) the fractional difference of the $\gamma$ 's. A practical case in our experience is a $25-\Omega(100-\Omega \mathrm{d}-\mathrm{c}$, nominal) platinum disk, which has been in use for close to 2 years to date; fractional differences in the resistances stayed constant at 0.4 percent, and in the $\gamma$ 's at 4 percent, which yield a possible error of 0.008 percent in voltage or current. A pessimistic figure of 0.01 percent from this source is used in the summation of the errors. In case of large fractional differences this error may be reduced by applying corrections. Also, a large difference in $\gamma$ 's may be compensated by reducing the difference of the resistances, and vice versa. Another way of avoiding this source of error is to make one half of the film out of a material having a relatively low $\gamma$ as compared with that of the other half $[12]$.

\subsection{Effect of Standing Waves on Voltage Measurement}

Assuming uniform diameters of the coaxial system, the error introduced in the voltage, $V_{x}$, applied at the input plane of an unknown voltmeter is obviously a function of (1) the VSWR, $S_{x}$, looking into its input port, and of (2) the distance, $x$, between the Bolovac film, $R_{m}$, and the above plane. This distance is 1 or 2 mils. High input impedance voltage monitors may have $S_{x}$ values of 200 at $1 \mathrm{GHz}$ [16]. The voltage ratio distribution in a lossless transmission line is given by

$$
\frac{V_{x}}{V_{m}}=\frac{1}{\cos \beta x+j \frac{Z_{0}}{Z_{x}} \sin \beta x} .
$$

For infinite input impedance of a voltmeter, this results in a possible error of 0.01 percent at $10 \mathrm{GHz}$ and of 0.02 percent at $20 \mathrm{GHz}$. It is therefore desirable to keep the distance between the two planes in question to a minimum.

\subsection{Effect of Resonant Tank Impedance Shunting $R_{m}$ on Current Measurements}

The input impedance, $Z_{s}$, of quarter wave, air dielectric, shorted, coaxial tuning stubs may be computed from [17]:

$$
Z_{s}=11.11(f)^{1 / 2} b F \frac{1}{n},
$$

where:

$f \equiv$ frequency in $\mathrm{Hz}$,

$b \equiv$ inner radius of outer conductor in $\mathrm{cm}$,

$F \equiv$ a constant found in figures 4 to 15 of the given reference,

$n \equiv$ odd number of quarter waves on the stub.

For $n=1$, at $1 \mathrm{GHz}$, and a $50-\Omega$ stub, with $b=0.64$ $\mathrm{cm}, Z_{s}$ is approximately $100,000 \Omega$ and the likely errors for $R_{m}$ values at 25,50 , and $100 \Omega$ are 0.025 , 0.05 , and 0.1 percent, respectively. One may use larger characteristic impedance stubs to reduce this error. For example, one may double the outer conductor radius and reduce the inner conductor radius to half of the $50-\Omega$ stub, and thereby reduce the corresponding above errors to $0.005,0.01$ and 0.02 percent.

Stubs may be used down to $50 \mathrm{MHz}$. At lower frequencies, lumped constant tanks may be preferable; obviously the highest possible $Q$ values with the lowest value tuning capacitors should be used. Errors in this case may be kept below 0.05 percent.

In either case the effect of the shunt impedance, $Z_{s}$, may be corrected for, once the value of $Z_{s}$ is determined.

\subsection{Effect of Standing Waves on Current Measurements}

Standing waves are at present a more critical source of error in current than in voltage measurements. The reason is the relatively large dimensions and high effective characteristic impedances of the present day current sensing devices. For example, so called 
"UHF" vacuum thermoelements are nearly always used at high frequencies. Bolometers, such as cylindrical resistors and themistors, have been used on rare occasions. Solid state thermoelements were recently developed for frequencies up to about $60 \mathrm{MHz}$, and bead type thermoelements were developed some years ago at the Bell Telephone Laboratories [18]. It is assumed that at the frequencies considered here, the current measuring devices are enclosed in a box approximating a coaxial structure with the current sensing element in series with the center conductor. Except for the case of thermistors and other beads (having diameters of the order of 0.1 to $1 \mathrm{~mm}$ ), the current sensing elements are long relative to the shorter wavelengths involved here. The UHF thermoelements have a glass envelope of about $2 \mathrm{~cm}$ in diameter and heater diameters starting at about 10 or $20 \mu$ (for the $5 \mathrm{~mA}$ current carrying capacity); the heaters are about $1 \mathrm{~cm}$ in length [19]. The uncertainties in calibrating these types of thermoelements are briefly discussed here as an illustration of the procedure in error evaluation.

Figure 11 shows a typical UHF feed-through type thermoelement mounted in a metallic tube. Planes $\mathrm{D}, \mathrm{H}$, and $\mathrm{A}$ are at the input end, middle, and output end of the heater, respectively. The Bolovac film is in plane C. The heater and its input and output leads form the center conductor of a coaxial system terminated by the Bolovac disk, $R_{m}$. This disk is, in turn, terminated by a negligible admittance. The effect of the discontinuities in the thermoelement coax system is negligible for the case at hand, at least
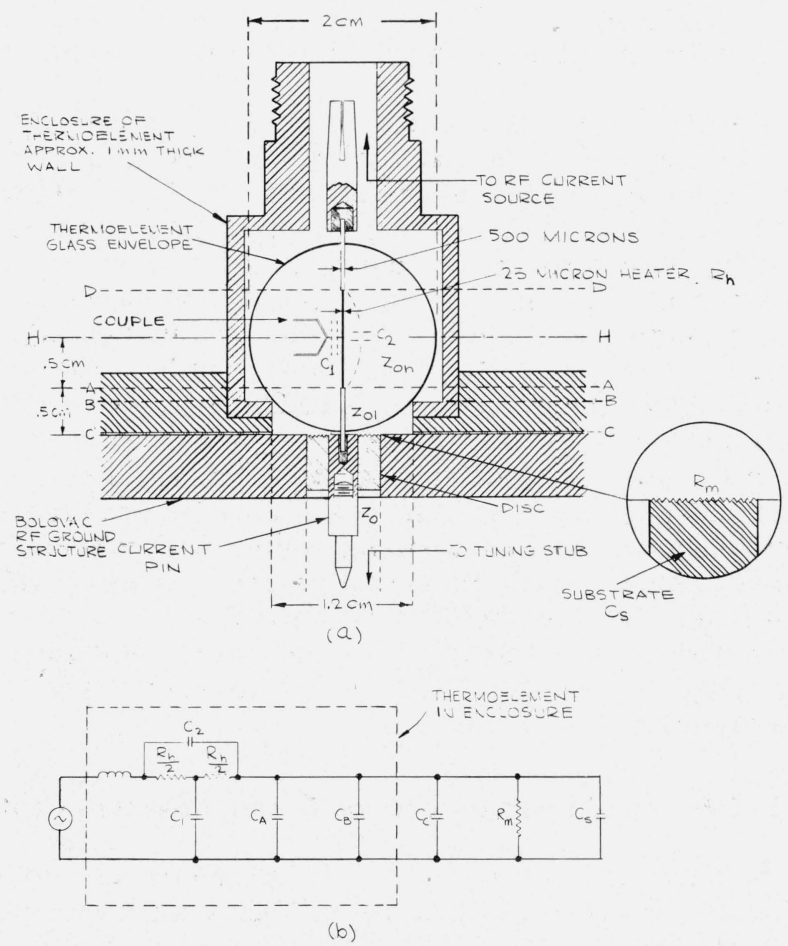

FigURE 11. UHF thermoelement under calibration by the Bolovac. Dimensions are approximate for use only as an illustration. to $5 \mathrm{GHz}$, as shown below. Current standing waves are present along the line formed by the heater $\left(Z_{0 \mathrm{~h}}\right)$ and the section formed by the output lead of the thermoelement $\left(Z_{01}\right)$. This second section is assumed to be uniform for the sake of simplicity. The heater section must be treated as a lossy line, because of the relatively high center conductor resistances, while the lead section may be considered lossless.

As previously indicated, the Bolovac-disk effective rf resistances may be of the order of $0.1 \Omega$ up to the value of $Z_{0}$. For the dimensions shown, $Z_{0 \mathrm{~h}}$ is approximately $400 \Omega$ and $Z_{01}, 220 \Omega$. It follows that for all resistance values of the Bolovac disk up to $200 \Omega$ there will be a current maximum in plane $C$.

Two effects of the standing wave need be considered in the heater, one caused by the nonuniform longitudinal distribution of the $\mathrm{rf}$ heater current, which may affect its temperature gradients. The other effect is the lower heater current as compared with that in planes $\mathrm{A}$ and $\mathrm{C}$.

To determine a pessimistic error versus frequency for the given physical dimensions, we apply the following well-known, low-loss, shorted (the higher the losses, the lower the effect) equation to the line section $Z_{0 \mathrm{~h}}$ :

$$
\frac{I_{x}}{I_{A}}=\cosh \alpha x \cos \beta x+j \sinh \alpha x \sin \beta x .
$$

Here $I_{x}$ and $I_{A}$ are the respective currents entering the planes at $x$ and $A ; x$ is the distance from plane $A$ toward the current source.

Assuming the case of a relatively low-loss line where the phase velocity $\beta$ is the same as in the air dielectric of the $Z_{0}$ line, the attenuation factor $\alpha$ introduced by heater resistances of the order of $10 \Omega$ or less is

$$
\alpha_{\mathrm{oh}}=\frac{r_{h}}{2 Z_{\mathrm{oh}}},
$$

where $r_{h}=$ heater resistance in ohms per meter.

For a typical $25 \mathrm{~mA}$ thermoelement, $r_{h} \cong 1,000 \Omega / \mathrm{m}$ and $Z_{\text {oh }} \cong 400 \Omega$, as stated above;

$$
\therefore \alpha_{0 \mathrm{~h}}=1.25 \text { nepers per meter. }
$$

Under these conditions the current distribution is, to a good approximation, the same as for a lossless shorted line, i.e.,

$$
\frac{I_{x}}{I_{C}}=\cos \beta x
$$

where $I_{C}$ is the rf current in the Bolovac disk and $x$ is the distance from $C$ towards the source. Direct computation of the impedances and current ratio for the dimensions given (at $1 \mathrm{GHz}$ and assuming $R_{m} \ll Z_{01}$ ), results in

$$
\frac{I_{A}}{I_{C}} \cong 0.995
$$




$$
\begin{aligned}
& \frac{I_{H}}{I_{C}} \cong 0.978 \\
& \frac{I_{D}}{I_{A}} \cong 0.956 .
\end{aligned}
$$

One may assume that the current is uniform along the heater and has a value of the current at the middle of the heater, or $0.978 I_{C}$. One can show that the error in this assumption will not exceed 0.2 percent. Based upon the distance between planes $\mathrm{H}$ and $\mathrm{C}$, one may therefore calculate the value of $I_{H}\left(I_{H}=0.978 I_{C}\right)$ with an estimated uncertainty of 0.2 percent. It is evident from eq (19) that this error will be lower as the heater resistance is increased.

One may also compute the top limit of $R_{m}$ for a given error for values above $200 \Omega$; in our experiments $R_{m}$ did not exceed $50 \Omega$. It also follows from the above that the length of the heater plus the leads seem to limit these thermoelements to frequencies below $1.5 \mathrm{GHz}$. Our observations and manufacturers data indicate the presence of resonances at frequencies above $1.5 \mathrm{GHz}$ for these thermoelements for the current ratings of 5 to $1,000 \mathrm{~mA}$; this is apparently a result of the residual inductance of the heater and of the shunt capacity across it. These thermoelements may therefore be used as indicators at frequencies above $1.5 \mathrm{GHz}$, but may be objectionable as calibrated instruments at higher frequencies because of likely instabilities.

It is, of course, apparent that the errors introduced by the standing waves are entirely avoidable when $R_{m}$ is made equal to the load impedance of the current sensor (in its enclosure) at its final application. This is so because the current entering the load will be calibrated for a given output of the couple and will be accurately reproduced irrespective of a possible difference between $I_{C}$ and $I_{H}$.

To summarize, for the above VHF thermoelements enclosed in 2-cm diam tubes and mounted at the Bolovac disk the error in assuming the corrected heater current equal to the Bolovac current will not exceed 0.4 percent at frequencies to $1 \mathrm{GHz}$; this error would not exceed 5 percent at $3 \mathrm{GHz}$, but the residual resonances may interfere above $1 \mathrm{GHz}$. For frequencies above $1 \mathrm{GHz}$ it seems best to use smaller current indicators. The source of error introduced by standing waves can be easily eliminated by using a value of $R_{m}$ sufficiently close to the intended load impedance of the thermoelement.

\subsection{Effect of Discontinuities on Current Measurements}

Figure 11 shows typical discontinuities as a result of steps in the nominally coaxial assembly of a thermoelement under calibration. The thermoelement may be enclosed in a metal box in such a way that the output-current lead length from the glass envelope of the thermoelement to the Bolovac disk is of the order of a millimeter. It is important to evaluate the effect of the shown discontinuities on current measurements because currents there are not con- tinuous. Equivalent circuits representing the effects of these types of step discontinuities have been comprehensively evaluated in the past [20]. The results there show that the discontinuities can be accounted for by placing a capacitor at the plane of the discontinuity, provided the transverse dimensions of the line are a small fraction of the wavelengths transmitted. The equivalent circuit of figure 1la is shown in figure $11 \mathrm{~b}$ omitting series inductances because these have been accounted for above in exploring the effect of the standing waves.

We consider the capacities $C_{1}$, between the heater and the thermocouple, and $C_{2}$, between the thermoelement leads,' a part of the thermoelement assembly; their effect is independent of the connection to the Bolovac and is lumped into the thermoelement assembly characteristics to be determined by the calibration measurements. $C_{A}$ in plane $A$, introduced by the discontinuity at the joint of the heater to the thermoelement output lead, is negligible; so is the capacity at the input end of the heater [20]. The capacitance $C_{s}$, contributed by the change in the dielectric medium, is lumped into the impedance terminating $R_{m}$ and thus becomes part of the quarter-wave stub tuner or of another tank. Therefore, it seems sufficient to evaluate the effect of the discontinuities in planes B and C.

The housing may enclose the thermoelement on all sides except for an opening at the current output; in this case the discontinuity in plane B is part of the housing and only the discontinuity in plane $\mathrm{C}$ is to be evaluated. Or the housing may be left open at the side in contact with the Bolovac; in this case there is no discontinuity in plane B. The equivalent capacities were looked up for both cases. $C_{c}$ turns out to be about $0.06 \mathrm{pF}$ for the first case of the complete enclosure and about $0.1 \mathrm{pF}$ for the second case. Because the capacitive current is in quadrature with the current in the disk, the error in the worst second case could be 1.4 percent at $5 \mathrm{GHz}$, with a $50-\Omega$ disk, and about 0.05 percent at $1 \mathrm{GHz}$. In the case of a complete enclosure the error at $5 \mathrm{GHz}$ would be reduced to about 0.5 percent. As the disk resistance is reduced, the error is reduced; for example, with a $10-\Omega$ disk, the error at $5 \mathrm{GHz}$ for a partial enclosure will be 0.05 percent.

A possible presence and effect of higher modes as a result of the above discontinuities is subject to further investigation. It is felt that the effect is negligible in our case, at least to $1 \mathrm{GHz}$; experimental evidence to that effect was obtained at $1 \mathrm{GHz}$. Two precalibrated thermoelements (in individual enclosures) were connected in tandem and the currents agreed well within the accuracies indicated. One must recall that the effect of suspected higher modes may always be reduced to any desired level by providing sufficiently long uniform line sections between the planes of the discontinuities and the reference plane of the current.

The conclusion is that a maximum enclosure of the thermoelement and a lowest practicable disk resistance will keep the error from this source to a negligible value even at $5 \mathrm{GHz}$. Reducing the physical 
size of the current sensor will further reduce this error. The case of the thermoelement is presented only as an example; effects of discontinuities may be similarly computed for other current sensors.

\subsection{Effects of Residual Impedances in the Ground System}

It was indicated above that the rf bypass capacitor $C_{b}$ (fig. 2) of the Bolovac may be a source of error because of its own impedance, particularly at or close to the frequency at which it may resonate with the distributed inductance of the assembly's ground system. $C_{b}$ may also be a source of higher modes. Figure 12 shows the likely equivalent circuit of this part of the system where $L_{1}$ and $L_{2}$ represent residual inductances of connecting bolts, of lumped capacitors, and ground circuits. $\mathrm{C}_{n}$ is the capacity between the insulated ground plates. Figure 12 a shows the equivalent circuit of a grounded rf source, the Bolovac (neglecting its residual ground inductances), and a grounded voltmeter being calibrated. Figure $12 \mathrm{~b}$ is the equivalent of figure 12a with the Bolovac ground plates insulated from each other. $L_{1}$ is the combined residual inductance of the bolts used to ground the center electrodes of the $1,000 \mathrm{pF}$ capacitors and of any other residual inductance of the capacitors. Figure $12 \mathrm{c}$ is the same as figure $12 \mathrm{~b}$ except the ground plates are interconnected with metal bolts and/or brackets having a total inductance of $L_{2}$. Figure 12d is the same as $12 \mathrm{~b}$ assuming an effective common laboratory ground which would normally short out $L_{2}$ but which generally cannot be realized at high frequencies. The two halves of the metal-film disks comprising $R_{m}$ are shown in figure $12 \mathrm{a}$ in series with their respective of bypassing capacities, 1/2 $C_{b}$. For

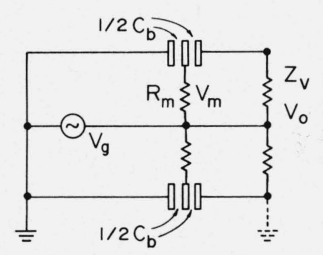

(a)

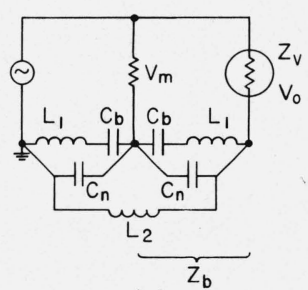

(c)

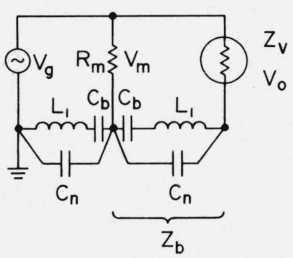

(b)

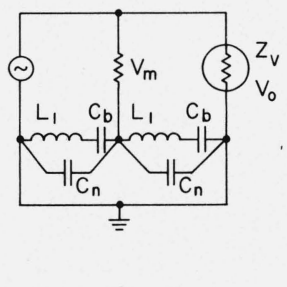

(d)
FIGURE 12. Circuits of Bolovac, RF source, and voltmeter under calibration.

(a) Equivalent circuit of setup when RF source and voltmeter have one terminal connected to common laboratory ground; residual inductances neglected. (b) Equivalent circuit with Bolovac ground plates insulated from each other; $L_{1}$ is residual inductance of $\mathrm{C}_{\mathrm{b}}$. (c) Same as (b) except ground plates are interconnected with metallic bolts and/or brackets. (d) Same as (b) assuming an effective common laboratory ground which generally cannot be realized at high frequencies. negligible $L_{1}, C_{b}=C_{b}^{\prime}+C_{n}$. The reactances of $C_{b}$ and the residual ground inductance on the input side may be considered a part of the impedance of the rf source and does not affect results as long as they remain sufficiently stable.

On the other hand, the impedance of $C_{b}$ in series with the output side of the disk may affect results. Observations indicated that the circuits shown in figure $12 b$ and $c$, and not $d$, are the likely equivalents at frequencies above, say, $100 \mathrm{MHz}$, where effect of the residual inductances becomes appreciable in the particular assembly tested. The input impedance, $Z_{v}$, of the device under calibration and the ground impedance, $Z_{b}$, appear as a voltage divider across the known voltage, $V_{m}$. $V_{0}$ should, therefore, be lower than $V_{m}$ by an amount depending upon the relative value of $Z_{b} / Z_{v}$. This may be appreciable at a frequency, $f_{r}$, at which the ground reactance elements are at resonance. With ground plates insulated from each other, such a resonance apparently did exist at approximately $550 \mathrm{MHz} . C_{b}$ of the core and auxiliary capacity ring was approximately 0.06 microfarads $(\mu \mathrm{F}) . V_{m}$ was greater than $V_{0}$ by several percent. It is evident from figure $12 \mathrm{~d}$ that if the common laboratory ground were effective at the high frequencies, and when both the generator and voltmeter were grounded, $V_{0}$ would be larger than $V_{m}$. This was not the case. With the outer ground plates noninsulated (i.e., interconnected with metal bolts), $f_{r}$ was about $300 \mathrm{MHz}$, and there were evidences of other resonances. Therefore, these plates were insulated from each other in our working assembly. In order to verify these deductions, a special voltage indicating device was constructed in such a way that it could be connected to the output of the Bolovac with and without the presence of the capacitor $C_{b}$ at the output. This voltmeter consisted of two half sections insulated from each other for dc, but common for rf. It was then calibrated with and without the output $C_{b}$ in the circuit to observe the ground effects. The only appreciable differences in the calibration values were at 550 and $300 \mathrm{MHz}$ indicated above.

A search of our models to date for resonances has not revealed any of an appreciable magnitude at frequencies to $8 \mathrm{GHz}$, except the one at about 550 $\mathrm{MHz}$ with the $C_{b}$ ring. The value of $C_{b}$ of the cores without the ring was approximately $2000 \mathrm{pF}$. This permitted the application of our cores without the $C_{b}$ ring down to frequencies of about $100 \mathrm{MHz}$ for the uncertainties cited above. Cores as well as rings with different values of $C_{b}$ may, of course, be readily designed depending on frequency ranges of interest. Small diameter cores with relatively large value of $C_{b}$ may be obtained with modern techniques, e.g., employing anodized electrode surfaces. Our cores used 1-mil thick mylar insulators.

Further experimental study of the effect of $C_{b}$ as a radial transmission line is perhaps in order. Available information on such a line [21] suggests that its characteristic impedance is fairly well approximated by the characteristic impedance of a coaxial line. The latter is assumed to have the same conductor separa- 
tion as the radial line and a mean radius equal to the mean radius of the radial line section. In our case this characteristic impedance in the Bolovac core would be of the order of 0.01 to $0.1 \Omega$. Despite such a low value of the characteristic impedance, the effective input impedance into the radial line section may still be appreciable at resonance frequencies. A possible way of eliminating this source of uncertainty seems to be to coat the disk surfaces of $C_{b}$ with some lossy compound, like carbon; this should reduce the input impedance of the radial section to a negligible value at resonance frequencies as well. As was stated before, an error may be introduced by the ratio of the input impedance of the radial line section to the input impedance of the voltmeter under calibration and not by the magnitude of the former.

It is readily apparent from figure 2 that measurements of rf currents are not affected by ground residuals nor by the value of $C_{b}$. The rf current enters the Bolovac disk from the center conductor, and its value is determined from the value of $V_{m}$ measured by the bridge. It returns via $C_{b}$ to the source. Therefore, the auxiliary capacitive ring is not essential for $\mathrm{rf}$ current measurement. It should thus prove relatively simple and economical to build Bolovacs for frequencies above, say, $100 \mathrm{MHz}$, particularly for practicable accuracies of 1 to 5 percent.

It is, of course, obvious that this error may be reduced to a negligible value even at resonance by making $C_{b}$ sufficiently large. However, this error can be avoided without the additional expense of increasing $C_{b}$ in one of three ways: (1) the core may be used without the ring so that $f_{r}$ may be materially shifted away from the desired calibration frequency; (2) a shift of $f_{r}$ may be obtained by decreasing $C_{b}$ of the ring by a sufficient amount, e.g., by breaking the contacts of some of the disk capacitors to ground; (3) $f_{r}$ may be shifted by temporarily shorting the ground plates of the ring. In the latter case, for a given $L_{2}$ and residual losses, the greater the value of $C_{b}$, the lower $f_{r}$ and the lower the value of the parallel resonance resistance and whence the lower the error in $V_{0}$.

Because it is rather difficult to predict residual parameters of a ground system and a likely $f_{r}$, it is best to search for such $f_{r}^{\prime}$ s experimentally whenever a new variation of the Bolovac is designed. For example, one or more coaxial outlets may be installed to observe the voltage $V_{b}$ across $C_{b}$, as we have done. The sharpness of a peak of $V_{b}$ as $V_{m}$ is kept relatively constant will indicate the presence of $f_{r}$, and the ratio of $V_{b} / V_{m}$ will indicate its possible detrimental effect. A measurement of $V_{b}$ will also indicate a possible error in $V_{0}$ in case the value of $C_{b}$ is too low for the lower end of the desirable frequency range. The likely error will, of course, be a function of the ratio of $Z_{b} / Z_{0}$; for example, at $3 \mathrm{MHz}$ for $C_{b}$ equal to approximately $0.06 \mu \mathrm{F}$, the reactance, $X_{b}$, is approximately $0.28 \Omega$, which is negligible compared to modern $\mathrm{rf}$ voltmeter input impedances. Even for a $50-\Omega$ resistive input impedance the error should not exceed 0.013 percent because the voltage drop across $X_{b}$ is in quadrature with that across $Z_{0}$. To search for $f_{r}$ and to measure $V_{b}$ it seems best to use good grade rf connectors with a voltmeter which will not introduce its own resonance effects. Once the values of $f_{r}$ and of $V_{b} / V_{m}$ have been found and steps determined to eliminate ground effect errors, these connectors may be removed or may be employed as bridge connecting terminals; however, their presence makes it possible to check the ground conditions at any time.

\subsection{Summary of Errors}

The uncertainties analyzed above may be conservatively summarized in percent of measured levels as shown in table 1 for voltage and table 2 for current. The uncertainties $1,2,3$, and 4 of table 1 hold for current measurements in typical enclosed present day UHF vacuum thermoelements. Three other causes are listed in table 2 . It is evident that wider ranges of voltage magnitudes and of frequencies may be realized with a given Bolovac at the sacrifice of some uncertainty.

TABLE 1. Summation of errors in voltage measurements

\begin{tabular}{|c|c|}
\hline Source & Percent \\
\hline 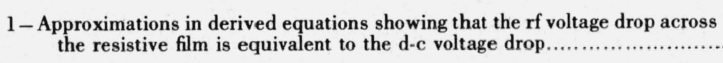 & \pm 0.00 \\
\hline $2-$ Magnitudes and instabilities of bridge components and biasing source.... & \pm .06 \\
\hline 3 -R.f source instability...... & \pm .01 \\
\hline 4-Quality of bolometric disk and substrate......... & \pm .01 \\
\hline 5 -Effect of standing waves on voltage at $10 \mathrm{GHz}$ (at $20 \mathrm{GHz}-0.020$ )... & \pm .01 \\
\hline 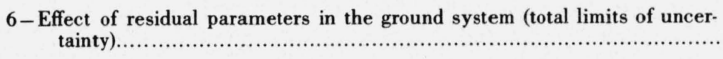 & \pm .00 \\
\hline Total limits of uncertainty for voltages of 0.1 to $5 \mathrm{~V}$ at $10 \mathrm{MHz}$ to $10 \mathrm{GHz}$.. & \pm .09 \\
\hline Same to $20 \mathrm{GHz} \ldots$ & \pm .10 \\
\hline
\end{tabular}

TABLE 2. Summation of errors in current measurements

\begin{tabular}{|c|c|}
\hline Source & Percent \\
\hline 1-4 Listed for voltage........ & \pm 0.09 \\
\hline 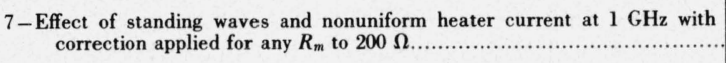 & \pm .40 \\
\hline Same, if $R_{m}$ is equal to the intended load of the thermoelement.... & \pm .00 \\
\hline 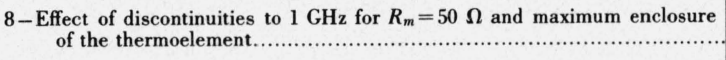 & \pm .05 \\
\hline Same for $R_{m}=10 \ldots \ldots$ & \pm .01 \\
\hline 9-Estimated average effect of resonant tank impedance shunting $R_{m} \ldots$ & \pm .05 \\
\hline Conservative total limit for frequencies to $1 \mathrm{GHz}$ and $R_{m} \leqslant 50 \ldots$ & \pm .60 \\
\hline Same for $R_{m} \cong$ intended load... & \pm .10 \\
\hline
\end{tabular}

\section{Comparison With Power Measurements}

It may be instructive at this point to discuss the overall uncertainties in measuring voltage and current in a plane $A$ arrived at in tables 1 and 2 as compared with the uncertainties of the power measurements 
against which the results are cited here. A thorough analysis of this issue is beyond the scope of this paper. Only the apparent major causes of the discrepancy are indicated as follows.

The power measurement uncertainties above $1 \mathrm{GHz}$ were \pm 1 to \pm 2 percent at best because a precalibrated bolometer power meter was used in this case. A calibration factor in terms of a calorimetric power standard was determined and the reading obtained with the meter was corrected accordingly. Direct comparison with the calorimetric power standard might have reduced the uncertainty to \pm 0.5 to \pm 1 percent. Because this was not yet done, the estimated uncertainty of our results of the voltage measurements is conservatively put at \pm 1 percent to $2 \mathrm{GHz}, \pm 2$ percent to $4 \mathrm{GHz}$, and \pm 4 percent to $8 \mathrm{GHz}$. These are the differences or disagreements, stated previously, between the Bolovac and the power-bolometer results.

Another reason for caution is the relatively small number of Bolovac disks tried so far in our laboratory. The differences between results of individual disks and the reproducibility of readings were within 1 percent. No effort was made to improve the bridge operation via a sufficiently careful calibration of its comporients to warrant the figures of uncertainty listed in the table.

The difference between determination of power transmitted along a transmission line employing voltage measurements as against other methods may be pointed out here. The multiple probe method of measuring power (and impedance) in TEM transmission lines was recommended years ago [22]. The reason for neglecting it might well have been the inability of measuring voltages with sufficient accuracy. With the latter difficulty remedied within the limits indicated here, it seems advisable to reconsider the method particularly for application in secondary standardization laboratories and field measurements. The method holds promise for better accuracies and greater economy. Power may be computed by measuring voltages at three probes spaced one-eighth wavelength apart. Only one voltage need be accurately known; the voltages at the other probes enter the equation simply as ratios to the first. It was shown above that the voltage in a given plane $A$, in a coaxial line, may be determined with an uncertainty of 0.09 percent. Assuming the uncertainty in the value of the characteristic impedance of presently available coaxial low-loss-precision line sections of \pm 0.05 percent, one can readily show that the power transmitted may be determined to an uncertainty of about \pm 0.2 percent over a wide range of standing wave ratios. This can be done without replacing the load of the system by a calorimeter, and indeed, without disturbing the system once probes have been installed and a voltmeter in combination with the probe section has been precalibrated for the purpose. Probe sections are needed for each frequency (or a band of frequencies with correction curves). However, the same voltmeter may be used for all frequencies. It may be one of a number of $50-\Omega$ input-impedance voltmeters or power meters presently available for frequencies up to $2 \mathrm{GHz}$ or higher. The voltmeter need not be permanently connected to the probe line section and can be used for other purposes in the laboratory or system installation.

The objective of this discussion is not to advocate voltage measurements in place of widely used power measurement techniques. The need of measuring power as a basic quantity is beyond dispute. However, one should be aware of the multiple probe method and its potentialities. An up-to-date survey of power measurements [23] lists \pm 2 percent as the lowest presently attainable uncertainty for feedthrough power meters at frequencies to $10 \mathrm{GHz}$. The lowest uncertainty in calibrating the effective efficiency of a bolometric power meter, using a calorimeter as the basic method, is \pm 0.2 percent. The latter involves complicated, relatively costly equipment, and has a long time constant. It would thus seem that the multiple probe method has a fair margin of safety in terms of uncertainty, particularly for use with field equipment and systems where accuracies of 1 percent are needed. Another advantage of the multiple probe method is the ability to measure impedances with the system in operation; an addition of a fourth probe makes this possible. Still other advantages are the relatively large voltage and power ranges that can be measured with one voltmeter and the relatively high decoupling factor, or low incident power absorbed by the probe and voltmeter.

\section{Conclusion}

Hopefully this paper, describing the Bolovac basically, will be followed by another on applicational steps, details, precautions, and additional results as well as on further perfection of the device. Throughout the development steps of the Bolovac, to date, the emphasis was on proving the validity and practicability of the principle and technique. No particular effort was made towards achieving optimum or potential accuracy, nor on establishing limits of voltage and current magnitudes, and a top limit of frequency. Considerable improvement is anticipated in the quality of the disks, particularly in their current-carrying capacity, sensitivity, and stability. Intercomparison with the dynamometer type ammeter [3] and with more accurate power measurements is pending. However, the results obtained so far amply illustrate the superior value of the Bolovac as an instrument for both high and medium accuracy calibrations of voltage, current, and possibly power meters over a wide frequency range.

Appreciation is hereby expressed to the Radio Standards Laboratory and to the NBS for the opportunity of developing the Bolovac; to P. A. Hudson, P. E. Werner and (the late) P. London for their splendid cooperation in disk developments; to W. J. Blank and R. P. Chariton for performing countless painstaking observations and measurements; to D. Holt for his assistance in the computation of the inductance of 
a short and wide rectangular film, and to C. M. Allred and C. C. Cook for critical and constructive reviews of the paper.

\section{Appendix}

One may reasonably question the effect of the imperfections of the film-resistor disk on the purity of the TEM mode. The nonuniformity of (1) the film thickness and of (2) the width of the annular ring may cause an effective residual inductance and thus introduce an error in the deduction that the disk impedance is purely resistive. A third possible source of uncertainty is the effective discontinuity introduced by the contact electrodes to the film. The likely limiting errors as a result of the above three imperfections will here be briefly analyzed.

The up-to-date state of the art on depositing resistive films and electrodes on substrate (preferably polished) surfaces indicates that the thickness uniformity of the film can be maintained to 1 to 2 percent over a $2 \mathrm{sq}$ in area [5, 22, 25]. Registration tolerances in the order of 0.0002 in $\left(5 \times 10^{-4} \mathrm{~cm}\right)$ of true position are obtained with photo-resist techniques [25]. The resistances of the two halves of the film (e.g., of platinum) may be made equal to 0.1 percent; the coefficient, $\gamma$, may be equal to within the same degree as the thickness (because $\gamma$ is a function of the thickness [13]). With tantalum or chromium on polished glass the $\gamma$ 's may be equal to within 0.1 percent. The tolerances are, naturally, a function of the resistance values desired; deposition rates can be controlled down to a few angstroms per minute [22]. Practical thickness limitations (at which the film characteristics approach those of the bulk of the material) vary widely with materials and are of the order of 50 to 200 angstroms [13].

Effect of film thickness variation on disk inductance. The area of the $7-\mathrm{mm}$ diam substrate over which the film is deposited is approximately $0.075 \mathrm{sq}$ in. On the basis of the above tolerances one may expect a thickness variation of about 0.03 percent. It will be assumed below that one half of the annulus differs in thickness from the other half, but each half is uniformly thick over its surface. To obtain a pessimistic evaluation, the effect of thickness differences of $0.03,0.1$, and 1 percent were considered.

To determine the effective residual inductance caused by the thickness differences one must compute the inductance of half an annulus (having the shape of half a washer) with radial currents, as shown in figure 13a. No analytical expression for the inductance of this case is available and the derivation of a suitable equation presents a problem beyond the scope of this paper. A conservative evaluation was therefore made on the basis of figure $13 \mathrm{~b}$. The film in figure $13 \mathrm{~b}$ is identical to that of figure 13a assuming an infinite radius of the coaxial line. It may be looked upon as a film resistor located in the transverse plane of a strip line with a center conductor and two outer "ground" conductors; the fringing fields are neglected. The

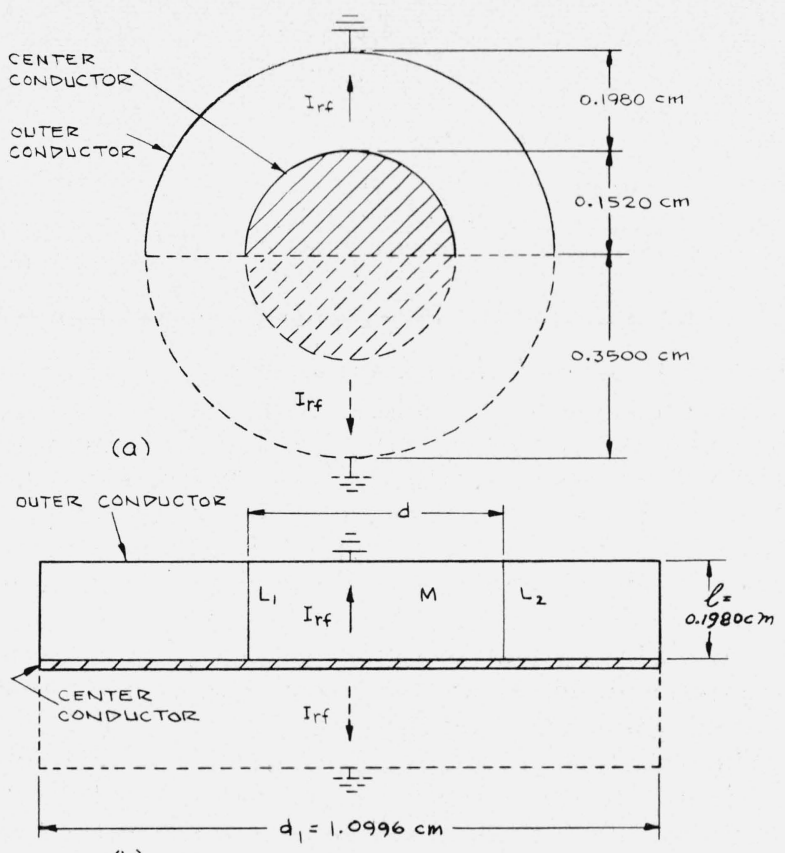

(b)

FIGURE 13. Bolovac coaxial transverse film and approximate equivalent film in strip line.

inductance of half of the film (between the center conductor and one of the outer conductors) can be computed for the transverse currents. Because of the dimensional configuration, conventional formulas derived for long conductors (for which $l \gg d$ ) cannot be used. Instead, the inductance was determined from an equation derived by Hoer and Love [26] using an electronic computer. Inductance values were computed for 1000-A thick films differing in thickness by $\pm 0.03,0.1$, and 1 percent. The inductance was $2.24908 \times 10^{-4}$ microhenries $(\mu \mathrm{H})$ for the 1000 -angstrom film. The differences in the inductance were as follows:

\begin{tabular}{c|c}
\hline \hline Thickness difference & Difference in $L$ \\
\hline & \\
Percent & Microhenries \\
+0.03 & $-9.39 \times 10^{-9}$ \\
-0.03 & $+1.06 \times 10^{-8}$ \\
+0.1 & $-3.20 \times 10^{-8}$ \\
-0.1 & $+3.42 \times 10^{-8}$ \\
+1 & $-6.60 \times 10^{-8}$ \\
-1 & $+3.43 \times 10^{-7}$ \\
\hline
\end{tabular}

One may show that the approach chosen is a pessimistic one as follows. Assume two conductive fibers (comprising two elements of the film) located a distance, $d$, from each other as illustrated in figure $13 \mathrm{~b}$. $L_{1}, L_{2}$, and $M$ are their respective self and mutual inductances. The combined inductance of the two filaments in parallel with currents flowing in the same direction is given by [27]. 


$$
L_{t}=\frac{L_{1} L_{2}-M^{2}}{L_{1}+L_{2}-2 M}
$$

here

$$
L_{1}=L_{2}=L
$$

and

$$
L_{t}=\frac{L+M}{2} \text {. }
$$

In the limit as $d \rightarrow \infty, M \rightarrow 0$, and $L_{t} \rightarrow \frac{L}{2}$. As $d \rightarrow 0$, $M \rightarrow K, L_{1} L_{2} \rightarrow K L, K \rightarrow 1$ and $L_{t} \rightarrow L$.

The presence of the mutual $M$ increases $L_{t}$. In the case of a cylindrical coaxial line (fig. 13a), the value of $M$ will always be smaller than for the corresponding filaments of case $13 \mathrm{~b}$ because of the relative angular positions of the filaments; indeed $M$ will be zero between filaments located at right angles to each other. Therefore $L_{t}$ will always be smaller in case 13a than in case $13 \mathrm{~b}$; so will the differences in $L$ as a result of differences in thickness. Therefore, the deductions for case 13b safely hold for case 13a.

The effective inductance of the entire annular film of 13a and of the top and bottom halves of $13 \mathrm{~b}$ is the difference between the inductances of the two halves and is equal to the figures given above for the differences in the thicknesses chosen.

The likely difference at the present state-of-the-art is of the order of 0.03 percent. For a $1000-\AA$ thick film the conservatively computed effective inductance might thus be as high as $1.06 \times 10^{-8} \mu \mathrm{H}$.

Again assuming a further pessimistic value of a thickness difference of one percent, the effective inductance may be as high as $3 \times 10^{-7} \mu \mathrm{H}$. This corresponds to a reactance of about $3.8 \times 10^{-2} \Omega$ at 20 $\mathrm{GHz}$. This quadrature component in series with, say, a $10-\Omega$ film may introduce an error of $7 \times 10^{-4}$ percent in the assumption that the $\mathrm{rf}$ impedance of the film is equal to its dc resistance. One should, perhaps, point out again that this is an "error" and in principle may be corrected for, if indeed the rf impedance can be measured with the required precision. The likely error may of course be computed for other film thicknesses and resistances. The higher the resistance of the film, the thinner it is and the higher may be the effective inductance. However, it enters as a quadrature component in the impedance. Thus, the overall error is not likely to be appreciable for any value of the film resistances and frequencies here under consideration.

Effect of variation of width of film. It was indicated above that the registration tolerances of the film are at present of the order of $5 \times 10^{-4} \mathrm{~cm}$ of true position. The incremental film width variation for a $7-\mathrm{mm}$, $50-\Omega$ coaxial line is thus approximately \pm 0.5 percent. The inductance for radial currents is approximately proportional to the width of the annulus (i.e., to the "length" of each elemental filament). Assuming in the limit that one half of the annulus differs in "length" from the other half by 0.5 percent and taking again the above example of the 1000-angstrom film having an inductance of $2.25 \times 10^{-4} \mu \mathrm{H}$, the effective inductance of the annulus might be about $11.25 \times 10^{-7}$ $\mu \mathrm{H}$. This might contribute an error of about the same magnitude as that of the difference in thickness, and again, a correction can be applied.

Effect of discontinuities introduced by contact electrodes. It was stated previously that registration tolerances may permit deposition of electrodes on the substrate to within $5 \times 10^{-4} \mathrm{~cm}$. Because of requirements associated with securing the position of the disk in the coaxial structure (e.g., by means of soldering, conductive epoxy cement, clampings, etc.), we will allow a conservative width of the electrodes on the disk surface of $15 \times 10^{-3} \mathrm{~cm}$. The computed [19] equivalent shunt capacity introduced by these electrodes in a $7-\mathrm{mm}, 50-\Omega$ line is approximately $15 \times 10^{-3} \mathrm{pF}$. The effect of the equivalent quadrature shunt reactance at $10 \mathrm{GHz}$ on current measurements employing a $50-\Omega$ film will be approximately 0.11 percent. With lower film resistances (most likely to be used for current standardization), the uncertainty is correspondingly lower. As was indicated previously, these discontinuities do not introduce uncertainties in voltage standardization; higher modes, if any, should be sufficiently attenuated along the uniform line sections normally present in input connectors to high frequency voltmeters.

\section{References}

[1] M. C. Selby and L. F. Behrent, A Bolometer bridge for standardizing radio-frequency voltmeter, J. Res. NBS 44, 15-30 (Jan. 1950) RP2055.

[2] Electrodynamic ammeter for very high frequencies, NBS Tech. News Bull. 34, No. 7, 103-104 (July 1950.)

[3] N. V. Frederick, An absolute electrodynamometer for high frequency currents (to be published).

Y. Aida, Research on the primary standard of current using short-circuited-ring for high frequencies, Researches of the Electrotechnical Laboratory, Japan, No. 639, pp. 1-93 (1963). (In Japanese).

[4] J. D. Ryder, Networks, Lines and Fields, Ch. 10, (Prentice Hall, Inc., New York, 1962).

[5] E. Keonjian, Microelectronics, pp. 181-191, 256, 257 (McGrawHill Book Co., Inc., New York, N.Y., 1963).

L. Braun and D. E. Lood, Precision thin-film cermet resistors for integrated circuits, Proc. IEEE 54, No. 11, 1521-1527 (Nov. 1966).

[6] A. E. Kennelly, Tables of Complex Hyperbolic and Circular Functions (Cambridge, Mass., Harvard University Press, 2d ed., 1927).

[7] J. J. Karakash, Transmission Lines and Filter Networks, pp. 116-125 (The Macmillan Co., New York, 1950).

T. Moreno, Microwave Transmission Design, p. 69 (Dover Publication, Inc., New York, 1958).

[8] M. C. Selby, Accurate radio-frequency microvoltages, Trans. of AIEE, Pt. 1, Communication and Electronics 72, 158-164 (May 1953).

R. D. Cutkosky, Evaluation of the NBS unit of resistance based on a computable capacitor, J. Res NBS (Phys. and Chem.) 65A, No. 3, 147-158 (May-June 1961).

[9] M. C. Selby, C. M. Allred, P. A. Hudson, I. S. Berry, High frequency power measuring bridge circuit, Pat. No. 2,833,620, (April 21, 1959).

[10] IRE Dictionary of Electronics Terms and Symbols. The Inst. of Radio Engineers, p. 54 (1961). For a comprehensive analysis of "imprecision," "systematic error," "uncertainty," etc., see: C. Eisenhart, Realistic evaluation of the precision and accuracy of instrument calibration systems, J. Res. NBS 67C (Eñgr. and Instr.) No. 2, 161-187 (Apr.-June 1963). H. H. Ku, Statistical Concepts in Metrology, Ch. 2, Handbook of Industrial Metrology, sponsored by Amer. Soc. of Tool and Mfg. Engrs. (Prentice Hall, Inc., New York. N.Y. 1967). 
[11] Reference Data for Radio Engineers, p. 129 (IT\&T Corp., New York, 4th ed., 1957).

[12] M. C. Selby, Single-bolometer series-parallel circuit for accurate voltage and power measurement, 19th Annual ISA Conference and Exhibit (Oct. 1964), Preprint No. 21, 6-1-61.

[13] W. Lewis, Thin Films and Surfaces, pp. 27, 69-71 (New York, Chemical Publishing Co., 1950).

[14] I. A. Harris, A coaxial film bolometer for the measurement of power in the UHF band, Inst. of Elec. Eng., Part B, 107, 67-72 (Jan. 1960).

[15] Glenn F. Engen, A dc-rf substitution error in dual-element bolometer mounts, IEEE Trans. on Instrumentation and Measurement, IM-13, Nos. 2 \& 3, 58-64 (June-Sept. 1964).

[16] M. C. Selby, W. J. Blank, and R. P. Chariton, Voltmeter calibration to $1 \mathrm{GHz}$, WESCON/65, Session 8, pp. 1-12.

[17] F. E. Terman, Electronic and Radio Engineering, pp. 106-107 (McGraw-Hill Book Co., Inc., 4th ed., 1955).

[18] C. G. Montgomery, Technique of Microwave Measurements, Radiation Laboratory Series No. 11, p. 189 (McGraw-Hill Book Co., Inc., New York, N.Y., 1947).

[19] M. J. O. Strutt and K. S. Knol, Measurements of currents and voltages down to a wavelength of 20 centimeters, Proc. IRE 2 7, No. 12, 783-789 (Dec. 1939).

[20] J. R. Whinnery, H. W. Jamieson and T. E. Robbins, Coaxial line discontinuities, Proc. IRE 32, No. 11, 695-709, (Nov. 1944).

[21] S. Ramo and J. R. Whinnery, Fields and waves in modern radio, 2d ed., pp. 395-401 (John Wiley \& Sons, New York, N.Y., 1953).

G. L. Ragan, Microwave transmission circuits, R. L. Series \#9, (1948), lst ed., pp. 100-114, 407-455.
[22] M. C. Selby, Voltage measurement at high and microwave frequencies in coaxial systems, proc. IEEE 55, No. 6, 877-882 (June 1967)

R. A. Schrack, Radio-frequency power measurements, NBS Circ. 536, p. 11 (Mar. 16, 1953).

D. D. King, Measurements at Centimeter Wavelength, pp. 197199 (D. van Nostrand Co., Inc., N.Y., 1952).

F. E. Terman and J. M. Pettit, Electronic Measurements, pp. 197-199 (McGraw-Hill Book Co., Inc., New York, N.Y., 1952).

M. Sucher and J. Fox, Handbook of Microwave Measurements, pp. 122-131 (Interscience Publishers, 3d ed., New York, 1963).

[23] A. Y. Rumfelt and L. B. Elwell, Radio Frequency Power Measurements, Proc. IEEE, 55, No. 6, 837-850 (June 1967).

[24] J. W. Nickerson and R. Moseson, Low-energy sputtering, Research and Development 16, No. 3, 53-57, (Mar. 1965).

[25] D. Hyndman, Basic thin film techniques, Electronic Design, pp. 86-93 (July 20, 1964).

[26] C. Hoer and C. Love, Exact inductance equations for rectangular conductors with applications to more complicated geometries, J. Res. NBS 69C (Phys. and Chem.) No. 2, 127-137, equations (14) and (19). (June 1965).

[27] F. Langford-Smith, Radiotron Designer's Handbook, 1953, 4th ed., RCA, Harrison, N.J., p. 148.

NBS Circular C74, Radio Instruments and Measurements, p. 18, (1937), Superintendent of Documents, Washington, D.C.

(Paper 72C1-269) 